\title{
Cosmic microwave background snapshots: pre-WMAP and post-WMAP
}

\author{
By J. Richard Bond ${ }^{1,2,4}$, Carlo Contaldi ${ }^{1}$, Dmitry Pogosyan ${ }^{3}$ \\ 1. Canadian Institute for Advanced Research Cosmology 83 Gravity Program, \\ Canadian Institute for Theoretical Astrophysics, McLennan Physical Laboratories, \\ University of Toronto, 60 St. George Street, Toronto, Ontario M5S 3H8, Canada \\ 2. Institut d'Astrophysique de Paris, 98bis Boulevard Arago, 75014 Paris, France \\ 3. Department of Physics, University of Alberta, \\ 412 Avadh Bhatia Physics Laboratory, Edmonton, Alberta T6G 2J1, Canada \\ 4. Institute of Astronomy, Madingley Road, Cambridge CB3 OHA, UK
}

Phil. Trans. R. Soc. Lond. A 361, 2435-2467 (2003). Published online October 15, 2003.

We highlight the remarkable evolution in the cosmic microwave background $(\mathrm{CMB})$ power spectrum $\mathcal{C}_{\ell}$ as a function of multipole $\ell$ over the past few years, and in the cosmological parameters for minimal inflation models derived from it: from anisotropy results before 2000; in 2000, 2001, from Boomerang, Maxima and the Degree Angular Scale Interferometer (DASI), extending $\ell$ to approximately 1000; and in 2002, from the Cosmic Background Imager (CBI), the Very Small Array (VSA), ARCHEOPS and the Arcminute Cosmology Bolometer Array Receiver (ACBAR), extending $\ell$ to approximately 3000, with more from Boomerang and DASI as well. Pre-WMAP (pre-Wilkinson Microwave Anisotropy Probe) optimal bandpowers are in good agreement with each other and with the exquisite one-year WMAP results unveiled in February 2003, which now dominate the $\ell \lesssim 600$ bands. These CMB experiments significantly increased the case for accelerated expansion in the early universe (the inflationary paradigm) and at the current epoch (dark energy dominance) when they were combined with 'prior' probabilities on the parameters.

The minimal inflation parameter set, $\left\{\omega_{b}, \omega_{c d m}, \Omega_{t o t}, \Omega_{\Lambda}, n_{s}, \tau_{C}, \sigma_{8}\right\}$, is applied in the same way to the evolving data. $\mathcal{C}_{\ell}$ database and Monte Carlo Markov Chain methods are shown to give similar values, highly stable over time and for different prior choices, with the increasing precision best characterized by decreasing errors on uncorrelated 'parameter eigenmodes'. Priors applied range from weak ones to stronger constraints from the expansion rate (HST-h), from cosmic acceleration from supernovae (SN1) and from galaxy clustering, gravitational lensing and local cluster abundance (LSS). After marginalizing over the other cosmic and experimental variables for the weak+LSS prior, the pre-WMAP data of January 2003 compared with the post-WMAP data of March 2003 give $\Omega_{t o t}=1.03_{-0.04}^{+0.05}$ compared with $1.02_{-0.03}^{+0.04}$, consistent with (non-baroque) inflation theory. Adding the flat $\Omega_{t o t}=1$ prior, we find a nearly scale invariant spectrum, $n_{s}=0.95_{-0.04}^{+0.07} \mathrm{com}-$ pared with $0.97_{-0.02}^{+0.02}$. The evidence for a logarithmic variation of the spectral tilt is $\lesssim 2 \sigma$. The densities are: for baryons, $\omega_{b} \equiv \Omega_{b} \mathrm{~h}^{2}=0.0217_{-0.002}^{+0.002}$ compared with $0.0228_{-0.001}^{+0.001}$, near the the Big Bang nucleosynthesis estimate of $0.0214 \pm 0.002$; for $\mathrm{CDM}, \omega_{c d m}=\Omega_{c d m} \mathrm{~h}^{2}=0.126_{-0.012}^{+0.012}$ compared with $0.121_{-0.010}^{+0.010}$; and for the sub- 
stantial dark (unclustered) energy, $\Omega_{\Lambda} \approx 0.66_{-0.09}^{+0.07}$ compared with $0.70_{-0.05}^{+0.05}$. The dark energy pressure-to-density ratio $w_{Q}$ is not well constrained by our weak + LSS prior, but adding SN1 gives $w_{Q} \lesssim-0.7$ for January 2003 and March 2003, consistent with the $w_{Q}=-1$ cosmological constant case. We find $\sigma_{8}=0.89_{-0.07}^{+0.06}$ compared with $0.86_{-0.04}^{+0.04}$, implying a sizable Sunyaev-Zeldovich (SZ) effect from clusters and groups; the high $\ell$ power found in the January 2003 data suggest $\sigma_{8} \sim 0.94_{-0.16}^{+0.08}$ is needed to be SZ-compatible.

Keywords: cosmology, particle physics, dark matter, dark energy

\section{The evolution of CMB spectra and cosmic parameters}

We have been in the midst of a remarkable outpouring of results from the CMB since 1999. The Royal Society Discussion Meeting focused on the eight pre-Wilkinson Microwave Anisotropy Probe (pre-WMAP) announcements made in 2002. The WMAP release was three weeks later, and a before-WMAP discussion without an afterWMAP discussion is unthinkable now. This paper applies the same methods of analysis to WMAP as to the earlier CMB experiments to put its singular forward step into context. In $\S 2$, we describe the different experiments that have contributed to the evolving picture. For more background material on methods and references, see Bond (1996), Bond \& Crittenden (2001), Bond et al. (2003a,b), Sievers et al. (2003), Ruhl et al. (2003) and Goldstein et al. (2003).

\section{(a) Grand unified spectra compared with WMAP}

Figure 1 shows how the CMB power spectrum, $\mathcal{C}_{\ell} \equiv \ell(\ell+1)\left\langle\left|T_{\ell m}\right|^{2}\right\rangle /(2 \pi)$ defined in terms of CMB temperature anisotropy multipoles $T_{\ell m}$, changed from the pre-WMAP determination shown at the Royal Society meeting using the data to January 2003 to the post-WMAP determination of March 2003; the agreement is excellent. How we got there is shown in figure 2, providing snapshots compared with WMAP for data that was available in January 2000, January 2002, June 2002, as well as January 2003. Accompanying this story is a convergence with decreasing errors over time on the values of the cosmological parameters given in figures 3,4 and table 4 .

Optimal spectra and their error matrices are calculated in exactly the same way that cosmological parameters are, with the parameters now the bandpowers $\mathcal{C}_{\mathrm{b}}$ in the chosen $\ell$-bins, b. Additionally, characterizing each experiment there are calibration uncertainties and often beam uncertainties, each adding additional parameters. Sample values for these are given in $\S 3 \mathrm{~b}$.

\section{(b) Basic parameters characterizing the early Universe and CMB transport}

Our philosophy has been to consider minimal models first, then see how progressive relaxation of the constraints on the inflation models, at the expense of increasing baroqueness, causes the parameter errors to open up. We adopt the basic set of seven cosmological parameters $\left\{\omega_{b}, \omega_{c d m}, \Omega_{\Lambda}, \Omega_{k}, n_{s}, \tau_{C}, \ln \mathcal{P}_{\Phi}\left(k_{n}\right)\right\}$ to facilitate comparison with results in Lange et al. (2001), Jaffe et al. (2001), Netterfield et al. (2002), Sievers et al. (2003) and Goldstein et al. (2003). How the values have converged upon the bull's-eye $2 \sigma$ determinations with WMAP is shown in figure 3 . 
In spite of the great success in extending the spectrum to high $\ell$, the evolution of the parameter errors was not that strong after January 2002 until WMAP. This is because the $\mathcal{C}_{\ell}$ model space is restrictive for inflation-based models, with high $\ell$ intimately related to lower $\ell$. On the other hand, when the experiments were treated individually (always with COBE-DMR), their $2 \sigma$ contours were all circling the bull's-eyes (Sievers et al. 2003).

The transport of the radiation through the era of photon decoupling is sensitive to the physical density of all of the species of particles present then, $\omega_{j} \equiv \Omega_{j} \mathrm{~h}^{2}$. We use two parameters, $\omega_{b}$ for baryons and $\omega_{c d m}$ for cold dark matter, to characterize this, but we should add $\omega_{h d m}$ for hot dark matter (massive but light neutrinos), and $\omega_{e r}$ for the relativistic particles present at that time (photons, very light neutrinos, and possibly weakly interacting products of late time particle decays). Here the latter is fixed for the conventional three species of relativistic neutrinos plus photons. The total matter density is

$$
\omega_{m}=\omega_{b}+\omega_{c d m}+\omega_{h d m} .
$$

Another two parameters characterize the transport from decoupling to the present, the vacuum or dark energy, encoded in a cosmological constant $\Omega_{\Lambda}$, and the curvature energy $\Omega_{k} \equiv 1-\Omega_{t o t}$. Of course $\Omega_{k}$ also determines the mean geometry. (When one wishes to focus on what the CMB can tell us about the nature of the dark energy, another parameter is often added, $w_{Q}=p_{Q} / \rho_{Q}$, where $p_{Q}$ and $\rho_{Q}$ are the pressure and density of the dark energy. If the vacuum or dark energy is reinterpreted as $\Omega_{Q}$, the energy in a scalar field $Q$ which dominates at late times, it would be likely to have complex dynamics associated with it. In that case, $Q$ and $w_{Q}$ would have spatial and temporal variations (except if $w_{Q}=-1$, the cosmological constant case). Spatial fluctuations of $Q$ are expected to leave a direct imprint on the CMB for small $\ell$. This complication is typically ignored, but should not be. It does depend in detail upon the specific model for $Q$.)

In this parameter space, $\mathrm{h}=\left(\sum_{j} \omega_{j}\right)^{1 / 2}$ and the age of the Universe $t_{0}$ are derived functions of the $\omega_{j}, \Omega_{k, \Lambda}$ and $w_{Q}$.

Another parameter is the Compton 'optical depth' $\tau_{C}$ from a reionization redshift $z_{r e h}$ to the present,

$$
\tau_{C} \approx 0.12\left(\omega_{b} / 0.02\right)\left(\omega_{m} / 0.15\right)^{-1 / 2}\left(\left(1+z_{\text {reh }}\right) / 15\right)^{3 / 2} .
$$

As long as $\tau_{C}$ is not too large, $\mathcal{C}_{\ell}$ is suppressed by a factor $\exp \left[-2 \tau_{C}\right]$ on scales smaller than the horizon at $z_{\text {reh }}$. For typical models of hierarchical structure formation, we expect $\tau_{C} \lesssim 0.3$. At the moment, even with WMAP, the CMB total anisotropy (TT) alone does not give such a constraint. It is the cross correlation of total anisotropy with polarization (TE) that leads to a detection (Kogut et al. 2003).

Two parameters characterize the early universe primordial power spectrum of gravitational potential fluctuations $\Phi$, one giving the overall power spectrum amplitude $\mathcal{P}_{\Phi}\left(k_{n}\right)$, and one defining the shape, a spectral tilt

$$
n_{s}\left(k_{n}\right) \equiv 1+d \ln \mathcal{P}_{\Phi} / d \ln k,
$$

at some (comoving) normalization wavenumber $k_{n}$. Instead of $\ln \mathcal{P}_{\Phi}\left(k_{n}\right)$, which is appropriate for connecting to early universe physics, we use as a basic amplitude variable $\ln \mathcal{C}_{10}$ when connecting to $\mathrm{CMB}$, and $\ln \sigma_{8}^{2}$ when connecting to LSS. 
To characterize inflation, even in the simplest models, we really need at least another two parameters, $\mathcal{P}_{G W}\left(k_{n}\right)$ and $n_{t}\left(k_{n}\right)$, associated with the gravitational wave $(\mathrm{GW})$ component. In inflation, the amplitude ratio $\mathcal{P}_{G W} / \mathcal{P}_{\Phi}$ is related to $n_{t}$ to lowest order, with $\mathcal{O}\left(n_{s}-n_{t}\right)$ corrections at higher order (e.g. Bond 1996). There are also useful limiting cases for the $n_{s}-n_{t}$ relation. With January 2003 data, and even with WMAP, the data are not powerful enough to determine much about the GW contribution, e.g. the WMAP team estimate the gravitational wave (tensor) contribution to be less than 0.72 of the scalar component in amplitude at the $95 \%$ CL.

As one allows the baroqueness of the inflation models to increase, one can entertain essentially any power spectrum. This implies a fully $k$-dependent $n_{s}(k)$ if one is artful enough in designing inflaton potential surfaces. The simple model

$$
n_{s}(k)=n_{s}\left(k_{n}\right)+\left[d n_{s}\left(k_{n}\right) / d \ln k\right] \ln \left(k / k_{n}\right)
$$

adds a logarithmic running index about a pivot scale $k_{n}$. As figure 1 and $\S 4$ indicate, this improves the fit to the data. It is also expected in inflation models, it is just a question of the size of the correction.

The tensor index $n_{t}(k)$ could also be a function, although it does not have as much freedom as $n_{s}(k)$ in inflation. For example, it is difficult to get $n_{t}(k)$ to be positive. One can also have more types of modes present, e.g. scalar isocurvature modes. The data have shown for quite a while that these would have to be subdominant relative to the scalar curvature modes, and would have to be even more so now.

Each experiment also contributes a parameter describing the uncertainty in the calibration, and possibly another for the uncertainty in the beam size.

\section{(c) CMB analysis pipelines: bandpowers to cosmic parameters}

In Gaussian models defined by a parameter set $\left\{y_{a}\right\}$, the probability distribution of the primary anisotropies is fully encoded in the isotropic power spectrum $\mathcal{C}_{\ell}\left(y_{a}\right)$ - as long as there is no preferred orientation (as might occur for small universes that are topologically nontrivial). The observed bandpowers for an individual experiment can then be tested against theoretical bandpowers $\mathcal{C}_{\mathrm{b}}\left(y_{a}\right)$, which are averages of the $\mathcal{C}_{\ell}$ 's over $\ell$-space 'window functions' $\varphi_{\mathrm{b} \ell}$ appropriate to the bands for the experiment in question. This represents a huge compression of the entire dataset and makes large model space computations feasible.

To use this information to estimate cosmological parameters, the entire likelihood surface as a function of the $\left\{\mathcal{C}_{\mathrm{b}}\right\}$ is needed with sufficient accuracy that the parameter estimations are not biased. It has been shown that individual bandpowers $\mathcal{C}_{\mathrm{b}}$ have distributions well characterized by a lognormal distribution in the variable $\mathcal{C}_{\mathrm{b}}+\mathcal{C}_{N \mathrm{~b}}$, where $\mathcal{C}_{N \mathrm{~b}}$ is an estimate of the noise in the band (Bond, Jaffe \& Knox 2000). The coupling between bandpowers is included as a weak correction, relying on the band-to-band correlations being relatively small - a demand imposed in the data analysis phase. What comes out are entropies, $S\left(y_{a}\right)$, i.e. log likelihoods. A slightly modified version of this prescription is used for WMAP (Verde et al. (2003); see also § 2e.)

There are two approaches to sampling the set $\left\{y_{a}\right\}$ that we have used. The main workhorse throughout our analyses up to January 2003 used fixed grids: a 
discrete set of parameter values are chosen a priori for six of the seven cosmic variables, with spacings in each of the dimensions designed by hand to be adaptively concentrated about the most probable values, but with sufficient spread to ensure that tails and multiple solution regions are well explored. The current database for the 'minimal inflation' parameter model contains $8.5 \times 10^{6}$ models, with dimensions $15 \times 13 \times 15 \times 12 \times 31 \times 11$ for the 'external parameter' set $\left\{\omega_{b}, \omega_{c d m}, \Omega_{t o t}, \Omega_{\Lambda}, n_{s}, \tau_{C}\right\}$, with edge cutouts requiring $\Omega_{m} \geq 0.1$.

The seventh (amplitude) parameter, $\ln \mathcal{C}_{10}$ or $\ln \sigma_{8}^{2}$, and the experimental calibration and beam uncertainty variables are continuous. They relax to their maximumlikelihood values, with errors characterized by the second derivative of the likelihood function. The number of these 'internal' continuous parameters may become much larger if we split the amplitude parameter into many, one for each band in $\ell$-space (or $k$-space if three-dimensional power spectra are the target). The shape $\mathcal{C}_{\ell}^{(s)}$ of an assumed spectrum multiplies the adopted window functions for the bands. For the optimal bandpowers that combine experiments together in figure $2, \mathcal{C}_{\ell}^{(s)}$ is usually varied to test robustness of the results, but an ensemble of external parameter models can be applied, e.g. in broken scale-invariance applications in $k$-space.

The first stage output is large entropy files that include maximum-likelihood values and Fisher matrices for the internal variables. These files are then picked up in postprocessing as various prior probabilities are applied, marginalizations are done, and one-dimensional (1D) and two-dimensional (2D) statistics are computed.

An advantage of a fixed grid is that it has allowed us to quickly check many prior cases and many experimental combinations, all on the same footing. Calibration uncertainties are handled either at the entropy stage with the complete experimental mix, or in postprocessing, since we know the amplitude distributions. When analysing an experiment, these operations are done again and again, as different hypotheses, band widths and positionings, estimation techniques, source removal methods, etc., are applied to the bandpowers for the experiments in question, so speed is essential. Combining DMR with the experiments was always a first step; now WMAP takes that role.

As new experiments are added which are qualitative improvements (like WMAP), errors may become smaller than grid spacings and further adaptivity of the grid is needed. This is so even with good interpolations and smoothing. We set a floor on parameter errors to be half the grid spacing of the encompassed grid: a value that was never reached before WMAP but has been with WMAP for a few parameters strongly bundled into the top few parameter eigenmodes and some priors.

The second approach is the Monte Carlo Markov Chain (MCMC) method (Metropolis et al. 1953; Christensen \& Meyer 2001; Christensen et al. 2001; Lewis \& Bridle 2002; Verde et al. 2003). It develops a set of independent chains, each a small (unstructured) grid on the parameter space that is constructed 'on the fly' rather than a priori. The elements of the chains are sampled according to welldeveloped MCMC algorithms designed to make the next step independent of prior ones. The spacing of the models computed changes with experimental combinations and priors adopted. As with the fixed-grid methods, some priors can be applied in postprocessing, which speeds up the procedure. These MCMC methods have now become feasible for CMB analyses because the $\mathcal{C}_{\ell}\left(y_{a}\right)$ computations with CMBfast (Seljak \& Zaldarriaga 1996) or CAMB (Lewis, Challinor \& Lasenby 2000) are effi- 
cient algorithmically, and are rapid enough on large numbers of models because of the remarkable speedup of individual computer processors in recent years. A nice Fortran 90 package is publicly available to do this (Lewis \& Bridle 2002). To do the statistics well, one needs not just many elements in a chain, but a number of chains. Thus it was really the advent of massively parallel machines that is allowing MCMC to become a major working tool for repetitive CMB and LSS analyses. For example, it is the method adopted by the WMAP team (Verde et al. 2003; Spergel et al. 2003), who applied it to the minimal six-parameter flat model, with the amplitude as an external parameter, and seven-parameter models with the $\Omega_{k}$, $w_{Q}, d n_{s} / d \ln k$ or $n_{t}$ allowed to vary in turn. They ran four chains and about 30000 models per chain to define their distributions. We have adapted Cosmomc to the parameter choices and ranges of our $\mathcal{C}_{\ell}$ database, and the many prior cases and experimental combinations used, to facilitate comparisons; e.g. $\tau_{C}$ can go out as far as 0.7 , which ensures likelihood drop-off to zero, but places sampling challenges. The major challenge for MCMC is to sample well the curved likelihood ridges at reasonable computational cost. Certain nonlinear combinations of our basic variables can help to straighten out the likelihood surfaces, in particular those with highly asymmetric errors, allow for more efficient and accurate computation, whether they be used in MCMC (Kosowsky et al. 2002; Chu, Kaplinghat \& Knox 2003; Verde et al. 2003) or for fixed or adaptive grids. For MCMC, another approach is to use variance matrices from small runs to make the steps efficient in the parameter space (Lewis \& Bridle 2002). For each experimental mix and prior, we use 16 chains run until convergence tests are satisfied for all of the variables. In spite of processor speed, the computations remain a challenge if many cases need to be run.

\section{(d) Weak, HST-h, SN1, LSS \& $\tau_{C}$ priors}

The parameter grids are chosen to be wide relative to conceivable cosmological models, yet are concentrated in the maximum-likelihood regions. The MCMC chains are allowed to vary over wide domains, and they automatically concentrate well. An important issue is the prior measure we impose upon the parameter space. Implicit in the adoption of a given variable set is that a uniform prior probability is chosen in each of the variables. If a variable is not well determined this can have a big influence (Lange et al. 2001).

We usually present the cosmological conclusions we draw from our analyses of the various $\mathrm{CMB}$ experiments using noncontroversial priors, ones that almost all cosmologists would agree to. Thus our standard weak one used in Lange et al. (2001) and subsequent works requires only $0.45 \leq \mathrm{h} \leq 0.90$ and $t_{0} \geq 10 \mathrm{Gyr}$. The addition of the flat prior has also become benign, thanks to the sharpness of the $\Omega_{k} \approx 0$ determination with the CMB rather than to the predilections of inflation theorists. (Although a major reduction in number of database models occurs when the flat $\Omega_{k}=0$ prior is applied, it is usually applied in the postprocessing phase.)

Data from sources other than the CMB can be incorporated as 'prior probabilities'. A stronger prior on the Hubble parameter, HST-h, uses an $h=0.72 \pm 0.08$ Gaussian distribution (Freedman et al. 2001). SN1 data imposes a prior in $\Omega_{\Lambda^{-}}$ $\Omega_{k}-w_{Q}$ space (Riess et al.1998, Perlmutter et al. 1999a,b, 2003). The CMB data apparently determine $\omega_{b}$ to higher accuracy than light-element-abundance obser- 
vations coupled to Big Bang Nucleosynthesis theory (Kirkman et al. 2003), hence applying a BBN prior is not of much interest.

The LSS prior we use (Bond \& Jaffe 1999; Lange et al. 2001; Bond et al. 2003b) also depends upon our parameter set. An important combination is the wavenumber of the horizon when the energy density in relativistic particles equals the energy density in nonrelativistic particles: $k_{H e q}^{-1} \approx 5 \Gamma_{e q}^{-1} \mathrm{~h}^{-1} \mathrm{Mpc}$, where $\Gamma_{e q}=$ $\Omega_{m} \mathrm{~h}\left(1.68 \omega_{\gamma} / \omega_{e r}\right)^{1 / 2}$. We represent the (linear) density power spectrum by a single shape parameter:

$$
\Gamma=\Gamma_{e q} \exp \left[-\left(\Omega_{b}\left(1+\Omega_{m}^{-1}(2 \mathrm{~h})^{1 / 2}-0.06\right)\right)\right]
$$

works reasonably well, to about $3 \%$ over the region most relevant to LSS; replacing $\Gamma$ by $\Gamma_{\text {eff }}=\Gamma+\left(n_{s}-1\right) / 2$ takes into account the main effect of spectral tilt over the LSS wavenumber band (Bond 1996). For low redshift clusters, the abundances determine a combination that is roughly $\sigma_{8} \Omega_{m}^{0.56}$ (with the degeneracy among the combination broken with high redshift cluster information). Weak lensing determines a similar combination.

With the wealth of data emerging from the Sloan Digital Sky Survey (SDSS) and the $2 \mathrm{dF}$ Redshift Survey (2dFRS), shape is a very powerful probe. However, the biasing of the galaxy distribution with respect to the mass becomes an issue if it is scale dependent, inviting caution - and for our purposes a weakened prior over what the data formally show. In the future, weak lensing should allow shape and amplitude to be simultaneously constrained without biasing uncertainties.

To be explicit, our prior for $\ln \sigma_{8}^{2}$ is of form $\sigma_{8} \Omega_{m}^{0.56}=0.47_{-0.02,-0.08}^{+0.02,+0.11}$, distributed as a Gaussian (first error) smeared by a uniform (tophat) distribution (second error). This straddles most of the values determined from weak lensing and many of those estimated from cluster abundances (shown in figure 5). We have also used a prior shifted downward by $15 \%$ to accommodate the lower values quoted for clusters in the literature. Our prior for the shape parameter is $\Gamma_{\text {eff }}=0.21_{-0.03,-0.08}^{+0.03,+0.08}$, which encompasses the recent SDSS and 2dFRS results as well as results from the Automated Plate Measuring (APM) angular survey and earlier redshift surveys. Fully embracing the 2dFRS galaxy power spectrum with a linear bias model to relate it to the total density power gives a stronger shape constraint, $\Gamma_{\text {eff }}=0.21_{-0.03,-0}^{+0.03,+0}$. Although some SDSS estimates are quite consistent, e.g. $\Gamma_{\text {eff }}=0.19_{-0.04,-0}^{+0.04,+0}$, different analysis methods and different datasets give wider ranges and the estimates do not incorporate possible complexities in the bias model. Thus, we have adopted a weak-LSS as opposed to a strong-LSS prior. Since $\Gamma \propto \omega_{m} / h$, with the improved CMB estimations of $\omega_{m}$ that arose in the January 2002 (and later) datasets, the shape constraint now has some similarity to an $h$ prior $(\S 4)$.

Values of $\Gamma_{e q}, \Gamma$ and $\sigma_{8}$ as estimated from the CMB data are given in table 4 . They are basically compatible with the LSS priors. One can get $\Gamma_{\text {eff }}$ from the $\Gamma$ and $n_{s}$ results in the table.

One of the most exciting results from WMAP was the TE cross-correlation of the E-mode of polarization and the total intensity $(\mathrm{T})$ at low $\ell$, interpreted as evidence for a $\tau_{C}=0.16 \pm 0.04$ detection, determined with a 'model independent' method by Kogut et al. (2003). The detection is not nearly as strong when ensemble-averaged over model space for the weak prior, as described in $\S 4$. The TE result is explicitly included in the Cosmomc treatment, but only TT is included in the $\mathcal{C}_{\ell}$ database 
used here. To incorporate the detection, we have constructed a $\tau_{C}$ prior, chosen to be broader than a $0.16 \pm 0.04$ Gaussian, $\tau_{C}=0.16_{+0.04,+0.06}^{+0.04,+0.06}$ in terms of Gaussian and top-hat errors. The MCMC results for $\tau_{C}$ we obtain when other parameters are marginalized is broader still, on both sides, and so we compare parameter estimates with and without this prior and find for most it makes little difference It does have an effect on marginalized amplitude determinations, in particular skewing somewhat the $\sigma_{8}$ distribution to higher values.

Sometimes there is 'tension' between the parameters estimated from CMB-only and those including non-CMB priors. This is extremely important to flag, since poor distribution overlap leads to smaller combined errors.

\section{(e) Degeneracy breaking \&5 parameter eigenmodes}

One is tempted to open up parameter space to a much larger set. There was a good reason for limiting the number in the pre-WMAP days: the spectra may not change much as the parameters vary, manifested by near-degeneracies among them. It is useful to disentangle the degeneracies by making linear combinations which diagonalize the error correlation matrix $\left\langle\Delta y_{a} \Delta y_{a^{\prime}}\right\rangle$, where $\Delta y_{a} \equiv y_{a}-\left\langle y_{a}\right\rangle$ and the averages are over the probability-weighted ensemble of models. These 'parameter eigenmodes' (Bond 1996; Efstathiou \& Bond 1999; Lange et al. 2001; Goldstein et al. 2003)

$$
\xi_{\alpha}=\sum_{a} \mathcal{R}_{\alpha a} \Delta y_{a} \text { obey }\left\langle\xi_{\alpha} \xi_{\beta}\right\rangle=\delta_{\alpha \beta} \sigma_{\alpha}^{2}
$$

The error on the eigenmode $\xi_{\alpha}, \sigma_{\alpha}$, is determined by the data and the priors. (Instead of $\Delta \omega_{b}$ and $\Delta \omega_{c d m}$, we use $\Delta \omega_{b} / \omega_{b}$ and $\Delta \omega_{c d m} / \omega_{c d m}$ in the combinations so their errors are relative and quantitatively meaningful relative to the other variables.)

Until the WMAP data, only four combinations of our seven could be determined within \pm 0.1 accuracy with the CMB (five with CMB+LSS), but with WMAP precision, for the March 2003 data, five can be determined (six with CMB+LSS), and two are determined to better than \pm 0.01 . Parameter eigenmodes arising from the current data are discussed further in $\S 4 \mathrm{~b}$.

Thus, WMAP precision gives us licence to open up the parameter space more. Here we only do this to a limited extent, by restricting ourselves to flat universes and replacing $\Omega_{t o t}$ by $w_{Q}$ or by $d n_{s}\left(k_{n}\right) / d \ln k$.

Both MCMC and fixed-grid approaches can have difficulty when the eigenmodes are precisely determined. Using variables which are nonlinear combinations of the $\left\{y_{a}\right\}$ motivated by the eigenmodes can aid with this, e.g. one characterizing the peak/dip pattern (the sound-crossing scale) and one the amplitude- $\tau_{C}-n_{s}$ neardegeneracy. Both degeneracies were exploited in limiting our $\mathcal{C}_{\ell}$ database storage requirements.

Expressing the LSS prior in terms of $\Gamma+\left(n_{s}-1\right) / 2$ and $\ln \sigma_{8}^{2} \Omega_{m}^{1.12}$ only (Bond et al. 2003b; Lange et al. 2001; Bond \& Jaffe 1999) is similar in spirit to keeping only the best determined 'eigenmode' from the redshift surveys and from the lensing or cluster surveys. However, the same mechanism that gives the acoustic peaks in $\mathcal{C}_{\ell}$ leads to oscillations in the density power for large $\omega_{b} / \omega_{m}$; i.e. further eigenstructure that would be revealed with high precision shape data. Similarly extra variables such 
as $\omega_{h d m}$ also lead to more eigenstructure. Higher redshift observations also break the $\ln \sigma_{8}^{2} \Omega_{m}^{1.12}$ near-degeneracy.

\section{(f) CMB pillars}

There were 'seven pillars' of the inflation paradigm that we were looking for in the CMB probe:

(1) the effects of a large scale gravitational potential at low multipoles;

(2) the pattern of acoustic peaks and dips;

(3) damping;

(4) Gaussianity (maximal randomness for a given power spectrum) of the primary anisotropies;

(5) secondary anisotropies associated with nonlinear phenomena, due to the SZ thermal and kinetic effects, inhomogeneous reionization, weak lensing, etc.;

(6) polarization, which must be there at the $c a .10 \%$ level, along with a specific cross-correlation with the total intensity;

(7) anisotropies and the associated polarization induced by gravity wave quantum noise.

At least five, and possibly six, of these have been seen. We have known about pillar 1 since COBE and FIRS, and found pillars 2 and 3 in the past few years, as discussed in $\S 3 \mathrm{c}, \mathrm{d}$.

Most, but not all, inflation models predict Gaussianity of the primary CMB fluctuations (pillar 4). This has been demonstrated to varying degrees with COBE, Maxima, Boomerang, the Cosmic Background Imager (CBI) and now with WMAP data. All secondary anisotropies and Galactic foregrounds will be non-Gaussian, so care must be taken in interpreting the inevitable deviations from Gaussianity.

The CBI, the Arcminute Cosmology Bolometer Array Receiver (ACBAR) and the Berkeley Illinois Maryland Array (BIMA) may have seen evidence for the thermal SZ effect, an aspect of pillar 5 (see $\S \S 2 \mathrm{~g}$ and $4 \mathrm{a}$ ).

Polarization (pillar 6 , see $\S 2 \mathrm{f}$ ), has been convincingly demonstrated. First there was the broadband detection by DASI of EE polarization and its TE crosscorrelation with total intensity, at levels consistent with inflation models. Then WMAP unveiled the TE cross-correlation spectrum to $\ell \sim 400$. The enhancement at $\ell \lesssim 20$ is the evidence for $\tau_{C}=0.16 \pm 0.04$ and an associated redshift of reionization $z_{\text {reh }} \sim 15$. The WMAP anticorrelation in TE observed at $\ell \sim 100$ is interpreted as proof that the dominant component of the perturbations giving rise to this effect is adiabatic.

Pillar 7 is an extreme experimental challenge, and some inflation models have gravity wave induced anisotropies too small for them ever be detected (see $\S 2 \mathrm{f}$ ).

\section{The CMB experiments}

\section{(a) January 2000}

We were dealing with upper limits on anisotropies until the 1990s. These limits were useful in ruling out broad ranges of theoretical possibilities. Then the familiar $2 \leq \ell \lesssim 20$ multipoles at the $30 \mu K$ level were revealed by COBE. This was followed in the years up to April 1999 by detections, and a few upper limits (ULs), at higher $\ell$ in 19 other ground-based (gb) or balloon-borne (bb) experiments. Some predated 
the 1992 COBE announcementin design and even in data delivery. We have the intermediate angle SP91 (gb), the large angle FIRS (bb), both with strong hints of detection before COBE, and then, post-COBE, more Tenerife (gb), MAX (bb), MSAM (bb), white-dish (gb, UL), argo (bb), SP94 (gb), SK93-95 (gb), Python (gb), BAM (bb), CAT (gb), OVRO-22 (gb), SuZIE (gb, UL), QMAP (bb), VIPER $(\mathrm{gb})$ and Python $\mathrm{V}(\mathrm{gb})$. Most had many fewer resolution elements than the 600 or so for COBE. One exception was SK95, which forged new ground compressing raw timestreams in software on to spatially extended 'pixels'. This heterogeneous dataset up to that time showed evidence for a peak (Bond, Jaffe \& Knox 2000), although it was not well localized. Improved first-peak localization occurred in summer 1999 with the Chile-based Toco experiment (Miller et al. 1999) and in November 1999 with the North American balloon test-flight of Boomerang (Mauskopf et al. 2000). Collectively we denote the results of all of these experiments as the January 2000 data. It pointed to $\Omega_{t o t} \sim 1$, but with broad errors (figure 3 ).

\section{Technical notes}

Boom-NA, Toco, QMAP, SK95, MSAM, SP94 and SP91 include quoted calibration errors; 13 bandpower detections from other experiments do not explicitly include them, but are often incorporated in the quoted error bars. The three bandpower upper limits use the 'equal variance approximation' to the likelihood (Bond, Jaffe \& Knox 2000), as opposed to the offset lognormal which is used for the rest. The low quadrupole is not included in the DMR bandpowers.

\section{(b) January 2002: Boomerang, Maxima and DASI}

In April 2000 dramatic results to $\ell \sim 600$ from Boomerang, the first CMB long duration balloon flight which circled Antarctica for 10.6 days in December 1998, were announced (de Bernardis et al. 2000; Lange et al. 2001). This was quickly followed in May 2000 by results from the night flight of Maxima (Hanany et al. 2000). Boomerang's best resolution was $10.7^{\prime} \pm 1.4^{\prime}$, about 40 times better than that of COBE, with tens of thousands of resolution elements. (The corresponding Gaussian beam filtering scale in multipole space is $\sim 800$.) Maxima had a similar resolution but covered an order of magnitude less sky. In April 2001, the Boomerang analysis was improved and much more of the data were included, delivering information on the spectrum up to $\ell \sim 1000$ (Netterfield et al. 2002). Maxima also increased its $\ell$ range (Lee et al. 2002).

Boomerang had six bolometers at $150 \mathrm{GHz}$ and 10 other bolometers at 90, 220 and $400 \mathrm{GHz}$. It mapped 1800 square degrees. The April 2000 analysis used only one channel and $440 \mathrm{deg}^{2}$. The April 2001 analysis used 4 channels at $150 \mathrm{GHz}$ and $800 \mathrm{deg}^{2}, 1.8 \%$ of the sky. These are the data used in the January 2002 and June 2002 sets (Netterfield et al. 2002). In December 2002, the final Boomerang-98 analysis was given (Ruhl et al. 2003), encompassing an area $1200 \mathrm{deg}^{2}, f_{\text {sky }}=2.9 \%$, using 3.5' pixels and the $150 \mathrm{GHz}$ channels. This is part of the January 2003 set.

Boomerang had a successful second long-duration balloon flight in January 2003, taking data for 13 days. The forecasts are for a well determined TT power spectrum even in the $\ell>1000$ regime, because the beam uncertainty is smaller than for the first flight. The $150 \mathrm{GHz}$ detectors were polarization sensitive bolometers of the 
sort that will be used on Planck, and polarizing grids were used with the 240 and $340 \mathrm{GHz}$ bolometers: good multi-band EE and TE spectra should emerge as well.

The South-Pole-based DASI (the Degree Angular Scale Interferometer) has 13 dishes of diameter $0.2 \mathrm{~m}$ and uses high-electron-mobility transistors (HEMTs) spanning 26-36GHz. An interferometer baseline directly translates into a Fourier mode on the sky. The dish spacing and operating frequency dictate the $\ell$ range. In DASI's case, the range covered is $125 \lesssim \ell \lesssim 900$. The total area covered was $288 \mathrm{deg}^{2}$ consisting of 32 independent maps of size $3.4^{\circ}$, the field-of-view. In April 2001, DASI unveiled a spectrum (Halverson et al. 2002) similar to that reported by Boomerang at the same time. The two results together reinforced each other and lent considerable confidence to the emerging (January 2002) $\mathcal{C}_{\ell}$ spectrum in the $\ell<1000$ regime.

\section{Technical notes}

For Maxima, 13 bandpowers, covering $36 \leq \ell \leq 1235$, were used, with a $4 \%$ calibration uncertainty and a $5 \%$ beam uncertainty. For both Boomerang sets, the power spectra use the 'Faster' method on $3.5^{\prime}$ pixels, the calibration error is $10 \%$ and the beam uncertainty is $13 \%$. Of the 25 bands, $2-21$ were used in our parameter analyses, covering $26 \leq \ell \leq 1025$. The other bands are marginalized. For DASI, the calibration uncertainty is $4 \%$, there is no beam uncertainty, and all 9 bands are used in our analyses.

\section{(c) June 2002: $C B I, V S A$ and BIMA}

The CBI (Cosmic Background Imager) is based 17000 feet above sea level on the Atacama Plateau in Chile. It has 13 dishes, $0.9 \mathrm{~m}$ in diameter, operating in the same HEMT channels as DASI. The instrument measures 78 baselines simultaneously. The larger dishes and baselines imply higher resolution, to $\ell$ of 3500 , a huge increase over Boomerang, Maxima and DASI. Only the analyses of data from the year 2000 observing campaign were reported in May 2002, covering three deep fields, of diameter $c a .0 .75^{\circ}$ (Mason et al. 2003), and three mosaic regions, each of size $c a .13$ $\operatorname{deg}^{2}$ (Pearson et al. 2003). Mosaics lace together multiple interferometer fields-ofview (FOVs) which overlap, allowing greatly improved resolution in $\Delta \ell$ over what many independent FOVs can give.

CBI data from 2001 roughly doubled the total amount available and increased the area covered. The combined two-year data are for three mosaic fields covering about $80 \mathrm{deg}^{2}$, including the two deep fields which overlap, and for one disjoint deep field. Preliminary results for the CBI 2000+2001 data were reported at the Royal Society meeting (January 2003). A full analysis is given in Readhead et al. (2003).

The Very Small Array (VSA) in Tenerife, also an interferometer operating at $30 \mathrm{GHz}$, covered the $\ell$ range of DASI, and confirmed the spectrum emerging from the Boomerang, Maxima and DASI data in that region in May 2002 (Scott et al. 2002). It is part of the June 2002 set. The VSA observed at longer baselines to increase its $\ell$ range and announced results in December 2002 (Grainge et al. 2003). It is included in the January 2003 set.

In June 2002, the interferometric millimetre array BIMA, operating instead at $30 \mathrm{GHz}$, announced power at $\ell \sim 6000$ had been found in random fields (Dawson et 
al. 2002). This is too far out in $\ell$ to be used for the analysis of primary anisotropies, but is important for secondary anisotropies (see $\S 2 \mathrm{~g}$ ).

\section{Technical notes}

The one-year CBI mosaic and deep field data use a conservative calibration error of $5 \%$. For parameters, bands 1-12 covering $\ell$ to 1940 are used in the odd binning of the mosaic power spectrum of Pearson et al. (2003). The 10 bands at higher $\ell$ are marginalized. When the deep field data are used in conjunction with the mosaic for optimal spectra, all bands are used. The deep data are not used for cosmic parameter estimation. The two-year data had a 3.3\% calibration error as of January 2003, but with WMAP's observation of Jupiter this could be reduced to $1.3 \%$, with a $3 \%$ recalibration downward in amplitude. The June 2002 VSA results have a calibration uncertainty of 3.5\%, and have 10 bands up to $\ell$ of 1000 . The January 2003 extended VSA results have the same calibration, and 16 bands extending to $\ell$ of 1700 . For March 2003 they were recalibrated with the lower calibration uncertainty, as for CBI.

\section{(d) January 2003: ARCHEOPS and ACBAR; Extended Boomerang, VSA 83 CBI}

The January 2003 data mix replaces Boomerang, the VSA and CBI by their extended sky results described in $\S 2$ b,c.

ARCHEOPS mapped $30 \%$ of the sky at a FWHM resolution of $15^{\prime}$ in a 22 hour balloon flight. It used the bolometer detectors and scanning strategy similar to those that will be employed for the Planck satellite. In October 2002, the ARCHEOPS team presented a power spectrum to $\ell=350$ that overlapped with the COBE/DMR power spectrum at low $\ell$ (Benoit et al. 2003), derived from $12 \%$ of the sky, one of their six $143 \mathrm{GHz}$ bolometers and one of their six $217 \mathrm{GHz}$ bolometers.

ACBAR was installed in January 2001 on the $2.1 \mathrm{~m}$ diameter Viper telescope at the South Pole. It has 16 bolometers in three frequency bands centered at 150, 220 and $280 \mathrm{GHz}$, with a best resolution of $c a .4^{\prime}$, allowing excellent coverage to high $\ell$. The analysis to obtain the power spectrum of Kuo et al. (2002) used the four bolometers at $150 \mathrm{GHz}$ available in the first observing season, the eight available in

the second, and covered $\sim 24 \mathrm{deg}^{2}$ in two fields consisting of correlated $\sim 3 \times 1.5$ $d e g^{2}$ patches. The cosmological parameters determined with its power spectrum in conjunction with other data were given in Goldstein et al. (2002). ACBAR is being further upgraded and continues to operate.

In Sept 2002, DASI announced the detection of polarization, and this is discussed in $\S 2 \mathrm{f}$ and as Pillar 6 .

\section{Technical notes}

The ACBAR set has a calibration uncertainty of $10 \%$ and an uncertainty of $3 \%$ on its $\sim 5.5^{\prime}$ beam at $150 \mathrm{GHz}$. For the analyses here, bands 2-14 are used, covering $300 \leq \ell \leq 3000$, with the first bin marginalized. (The window function of the first bin is oscillatory and reaches reasonably high $\ell$. However, for optimal spectra and parameter results in Goldstein et al. (2003), the first bin has also been used, and yields similar results to those obtained without it, as described below.) ARCHEOPS has a calibration uncertainty of $7 \%$ and an uncertainty of $10 \%$ on its $15^{\prime}$ beam. Sixteen bands covering $15 \leq \ell \leq 350$ are used. 
(e) March 2003: WMAP

In June 2001, NASA launched the five-frequency all-sky HEMT-based WMAP satellite. The best resolution of $12.6^{\prime}$ at $94 \mathrm{GHz}$ corresponds to a 'Gaussian' beam size of $\ell \approx 640$. The first-year results unveiled in February 2003 were as spectacular as forecast, definitive through the second peak. The noisy error bars at higher $\ell$ will subside as the observing period increases, and the beam is very well known so that we may expect good spectra out to $\ell \sim 1000$ with four years of data.

COBE was fundamental to every parameter determination with pre-WMAP data. The WMAP verification that the COBE/DMR maps were accurate in detail was by itself an important step. Pillar 1's Sachs-Wolfe effect dominates at low $\ell$. It includes both the 'ordinary' effect from fluctuations in $\Phi$ on the last scattering surface and the 'integrated' Sachs-Wolfe (ISW) effect, from the change of $\Phi$ with time so the energy of a photon climbing out of a potential well differs from that when it dropped in. Other effects can influence low $\ell$, the contribution from tensor modes, and more exotic possibilities involving low mass scalar fields, modified topologies, radically broken scale invariance, etc. For the best-fit $\Lambda \mathrm{CDM}$ models, an upturn in $\mathcal{C}_{\ell}$ from the ISW is predicted, but instead a downturn is observed with WMAP, confirming and extending the puzzles associated with the relatively low quadrupole. It has been our practice to drop the $\ell=2$ mode in parameter estimation (marginalize over it), and we continue it here, but recognize it could be pointing to new physics. The quadrupole has such large cosmic variance that including it or not does not change parameter determination in the minimal inflation models much.

The low- $\ell$ DMR data has been used to constrain the size of the Universe, basically from the scale of its hot and cold spots. If our manifold was much smaller than the apparent distance to the last scattering surface, there would not be large-scale spots in DMR maps, and smaller scale ones would be images of each other, with placement and details dependent upon specifics of the manifold. The complication here is that there are many possible manifolds, and each has an orientation, so it is possible to find universes that are just big enough to be more probable than the conventional inflation models with far fewer degrees of freedom in which the large-scale hot and cold spots are not geometrically correlated as in the topology case. See Bond, Pogosyan \& Souradeep (2000) for a discussion. WMAP data should allow more powerful tests and improve the current constraints on size somewhat.

\section{Technical notes}

The WMAP power spectra for our analyses are those obtained in Hinshaw et al. (2003) for TT to $\ell=900$ and in Kogut et al. (2003) for TE to $\ell=512$, using the construction in Verde et al. (2003) for the correlated errors.

For our $\mathcal{C}_{\ell}$ database approach, we include the $0.5 \%$ calibration uncertainty, use the offset lognormal approximation to the likelihood surface, and, as for DMR, we marginalize over the WMAP quadrupole. We have compressed the data to speed up our analyses. We have used the full 899 individual multipoles of Hinshaw et al. (2003) and Verde et al. (2003), including correlations out to a (tiny) sideband cutoff. Doing many large matrix multiplies can be slow, as relaxation to the amplitudes is done for each of the 8.5 million model elements in the database. Although these operations could be sped up considerably by approximate log-likelihood estimates 
to reject extremely improbable models, and there are many of these, we have so far used brute force through all models.

We have also used compressions of the WMAP data onto fewer bands for parameter estimations, optimal spectra and calibration estimation. For example, a 98-band compression of the data preserves all signal and noise information and side-band correlations in the bands. has $\Delta \ell$ spacings ranging from 1 at small $\ell$, to 7 to 5 to 7 through $\ell=373,9$ through 565 , with a gradual increase beyond 600 , and only 4 broad bands beyond 700 (with relative errors above $50 \%$ ). A best fit shape was used in the compression, but other choices, including a flat shape, give very similar spectra where WMAP dominates the data. In a 49-band compression, the band spacings are essentially doubled, and in a 19-band compression, the Boomerang $\Delta \ell=50$ spacing was used. Parameter results using the $\mathcal{C}_{\ell}$ database are quite consistent among the 899-band, 98-band and 49-band sets, with slightly sharper errors with the 899-band set. We do find small (sub- $1 \sigma$ ) deviations in parameters that are bundled into some of the exquisitely determined eigenmodes for the 899 set compared with the 98 set.

For our MCMC calculations, the WMAP likelihood routines of Verde et al. (2003) are adopted, since these are used in the Cosmomc package (Lewis \& Bridle 2002). This uses a hybrid of an offset lognormal and a Gaussian distribution to compute parameters. The quadrupole is included. The small calibration error of $0.5 \%$ is not. For $\mathcal{C}_{\ell}$ construction, CMBfast was used for the database and CAMB is used in Cosmomc. Other differences between the database and MCMC treatments of WMAP are bigger, e.g. mimicking the effects of the TE data with a $\tau_{C}$ prior. The parameter results for the 98-band case using MCMC are in good agreement with those using the database.

\section{(f) Beyond March 2003: Planck and targeting polarization}

Many other CMB experiments on the ground and in balloons will happen before the European Space Agency's (ESA's) Planck satellite is launched in 2007. Planck combines bolometers, many of which will be polarization sensitive, and HEMTs. Its best resolution of $\sim 5^{\prime}$ and detector sensitivity should allow the damping tail and power spectrum modifications due to secondary effects, including weak lensing, to be very well determined. The all-sky component-separated intensity and polarization maps will be superb for interstellar matter and extragalactic source research as well as cosmological research. Such accuracy is needed to open up the cosmic parameter space and search for anomalies that may signal new physics beyond the minimal inflation concordance model we are drawn to now.

Polarization is described by a $2 \times 2$ tensor on the sky, with the components related to the four Stokes parameters, $T, Q, U, V$, with $T$ the total intensity (i.e. temperature). The polarization dependence of Compton scattering induces a welldefined polarization signal emerging from photon decoupling, arising from the quadrupole nature of the viscosity-induced anisotropic stress tensor. For primary CMB fluctuations, circular polarization is not there and $V$ vanishes. When $Q$ and

$U$ maps are Fourier transformed and are rotated into a basis related to the angular wavevector, these give $E$-type (grad) and $B$-type (curl) 'maps'. Scalar perturbations in linear theory do not generate $B$-type modes, so their absence is a check. 
Tensor perturbations generate both, and detection of $B$-modes at low $\ell$ would be a direct signature of a gravitational wave background (pillar 7).

Given the total $\mathcal{C}_{\ell}^{(T T)}$ of figure 1, we can forecast the polarization power $\mathcal{C}_{\ell}^{(E E)}$

and cross-correlation power $\mathcal{C}_{\ell}^{(T E)}$ : the maximum signal is expected at $\ell \sim 900$, with amplitude $\sim 5 \mu \mathrm{K}$ over $\ell \sim 400-1600$.

The great race to first detect $\mathrm{CMB} E$-mode polarization was won by DASI (Leitch et al.2002, Kovac et al. 2002), with 271 days of polarization data on rwo deep fields $\left(3.4^{\circ} \mathrm{FOV}\right)$ showing a $5 \sigma$ detection with a value $0.8 \pm 0.3$ of the forecasted amplitude from $T$ for inflation-based models. The cross-correlation of the polarization with the total anisotropy had an amplitude $0.9 \pm 0.4$ of the forecast. These detections used a broadband shape covering the $\ell$ range $\sim 250-750$ derived from the theoretical forecasts. The powerful cosmological implications of the remarkable WMAP TE bandpowers have already been discussed.

Forecasts indicate solid EE power spectrum determinations are likely soon from the ongoing CBI polarization observations and Boomerang's January 2003 flight with polarization-sensitive bolometers. Both are optimally sensitive to the $\ell \sim 900$ region where the EE power is expected to peak. MAXIMA will fly again as the polarization-targeting MAXIPOL. Other EE experiments, operating or planned, include AMiBA, CAPMAP/PIQUE, COMPASS, CUPMAP, POLAR, Polarbear, Polatron, QUEST and Sport/BaRSport, among others. We are also awaiting WMAP's EE results.

Although the strength of the $B$-mode induced by gravity waves is model specific, the amplitude is expected to be quite small even at low $\ell$. Nonetheless there are experiments such as BICEP being planned to go after $\mathcal{C}_{\ell}^{(B B)}$ in these low $\ell$ ranges. Planck could also make such a detection. A nice figure summarizing EE and BB bandpower forecasts for various experiments is given in Hivon \& Kamionkowski (2002). The promise is sufficiently exciting that a CMBPol satellite is being contemplated by NASA as the next step in space for the CMB after Planck.

\section{(g) Beyond March 2003: targeting secondary anisotropies}

Spectral distortions from the CMB black body must exist as a result of nonlinear processes and will have associated anisotropies. The spectrally well-defined SZ distortion associated with Compton upscattering of CMB photons from hot gas has not been observed in the spectrum. The COBE/FIRAS $95 \%$ upper limit of $6 \times 10^{-5}$ of the energy in the CMB is compatible with values up to around $10^{-5}$ expected from the cosmic web of clusters, groups and filaments in the inflation-based class of models considered here, and places strong constraints on the allowed amount of earlier energy injection, e.g. ruling out mostly hydrodynamic models of LSS.

The SZ effect has been well observed at high resolution with very high signal-tonoise along lines-of-sight through a large number of clusters now, by single dishes, the OVRO and BIMA millimetre arrays, and the Ryle interferometer. This tells us, among other things, that the CMB comes from further away than redshift $z \sim 1$ - if we had any residual doubt. The SZ effect in random fields may be responsible for the power at $\ell>2000$ seen in the CBI deep data (figure 3) (Mason et al. 2003; Bond et al. 2003b), in the BIMA data at $\ell \sim 6000$ (Dawson et al. 2002), and possibly in the ACBAR data (Goldstein et al. 2003). Multi-frequency observations 
to differentiate the signal from the CMB primary and radio source contributions will be needed to show this.

A number of planned HEMT-based interferometers are being built with this ambient effect as a target: CARMA (OVRO+BIMA together), the SZA (from Chicago, and to be incorporated in CARMA), AMI (based in Britain, including the Ryle telescope) and AMiBA (from Taiwan). Bolometer-based experiments will also be used to probe the SZ effect, including: the CSO (Caltech Sub-Millimetre Observatory, a $10 \mathrm{~m}$ diameter dish) with BOLOCAM on Mauna Kea; the LMT (Large Millimetre Telescope, with a $50 \mathrm{~m}$ diameter dish) in Mexico; APEX, a $12 \mathrm{~m}$ diameter German single dish based in Atacama; Kobyama, a 10 m diameter Japanese single dish; and the $100 \mathrm{~m}$ Green Bank telescope. Large bolometer arrays with thousands of elements and resolution below $2^{\prime}$ are also under development: the South Pole Telescope (SPT, Chicago) and the Atacama Cosmology Telescope (ACT, Princeton).

The kinetic SZ effect due to the motion of clusters in the low-to-moderateredshift cosmic web, or just clumpy ionized gas in the high redshift cosmic web, has the same spectral signature as primary anisotropies, and so is harder to disentangle.

The (non-Galactic) distortions from the black body that have been detected in the COBE FIRAS and DIRBE data are associated with starbursting galaxies due to stellar and accretion-disk radiation downshifted into the infrared by dust then redshifted into the sub-millimetre. This background has energy about twice the total of that in optical light, about a tenth of a percent of that in the CMB. About $50 \%$ of this sub-millimetre background has been identified with sources found with the SCUBA bolometer array on the JCMT. Anisotropies from dust emission from these high redshift galaxies are being targeted by the JCMT (with a SCUBA-2 very large array in development), the OVRO millimetre interferometer, the CSO, the SMA (Sub-Millimetre Array) on Mauna Kea, the LMT, the ambitious US/ESO ALMA millimetre array in Chile, the LDB BLAST, and ESA's Herschel satellite.

\section{CMB analysis and phenomenology}

\section{(a) CMB pipelines: from timestreams or visibilities to bandpowers}

Analysing CMB experiments involves a pipeline that takes the raw detector timestreams or visibilities from correlators for interferometers, flags and cleans them, and usually generates maps, from which bandpowers and higher order statistics are derived, ideally after separating component signals by using their differing frequency and spatial dependences. The step from bandpowers to cosmic parameters described in $\S 1 \mathrm{c}$ may be the goal, but it is not where most of the time is spent. Indeed parameter determination is used as a diagnostic along with everything else as the CMB teams struggle to understand in detail their experimental results. Every new round of data generates a fresh look at pipelines, and often new faster algorithms for proceeding. Recent pipelines are described in e.g. Bond \& Crittenden (2001), Netterfield et al. (2002), Hivon et al. (2002) and Ruhl et al. (2003) for single-dish bolometers, in e.g. Myers et al. (2003) for interferometry, and in Bennett et al. (2003), Hinshaw et al. (2003) and Verde et al. (2003) for WMAP.

For single-dish experiments, the timestreams are turned into spatial maps for each frequency: an average temperature in each pixel and a pixel-pixel noise correlation matrix from which the bandpowers, noise in the bandpowers and band-band 
error matrices are derived. The first step is to extract the sky signal from the noise, using the only information we have, the pointing matrix mapping a bit in time onto a pixel position on the sky. In the analysis of Boomerang, and subsequent work including for WMAP, powerful use of Monte Carlo simulations was made to evaluate the power spectrum and other statistical indicators in maps with many more pixels than was possible with the conventional matrix methods described in Bond, Jaffe \& Knox (1998) and Borrill (1999).

For interferometer experiments, the basic data are visibilities as a function of baseline and frequency, with contributions from random detector noise as well as from the sky signals. A baseline is a direct probe of a given angular wavenumber vector on the sky, and hence suggests that we should make 'generalized pixel' maps in 'momentum space' (i.e. Fourier transform space) rather than in position space, as for Boomerang. For $\mathrm{CBI}$, the $>\mathcal{O}\left(10^{5}\right)$ visibility measurements of each field were 'optimally' compressed into a $<\mathcal{O}\left(10^{4}\right)$ coarse-grained lattice in momentum space from which the power spectrum was calculated using matrix methods (Myers et al. 2003).

The important step of separating multi-frequency timestream data into the physical components on the sky is fundamental, still under active development, and will remain so for a long time, as our precision increases. The sources are the primary $\mathrm{CMB}$, the thermal and kinetic SZ effects, the dust, synchrotron and bremsstrahlung Galactic signals, the extragalactic radio and sub-millimetre sources, possibly spinning dust, and of course the sources we have not thought of yet. An example is the treatment of point sources: at the high resolution of CBI and its 30 $\mathrm{GHz}$ frequency, the contribution from extragalactic radio sources is significant, so known point sources were projected out of the visibilities by using a large number of constraint-template matrices which marginalize over all affected modes, using positions from the $(1.4 \mathrm{GHz})$ National Radio Observatory Very Large Array Sky Survey (NVSS) catalog (Mason et al. 2003). Even though the WMAP resolution is substantially lower than that of CBI, WMAP cut out some 700 sources in its analysis. Boomerang cut out three quasars.

For extended sources, the most expedient method is to just cut out problematic areas, e.g. with Galactic latitude cuts to remove emission from the plane, as was done for DMR, WMAP and Boomerang. Extensive use is also made of templates that come from Galactic observations, e.g. the IRAS/DIRBE maps of sub-millimetre emission, HI maps, etc., and from the experiments themselves, with the highest and lowest frequency maps often being completely dominated by foregrounds (as for the dust-dominated $400 \mathrm{GHz}$ Boomerang map). Having errors on primary signal maps that reflect the residual contamination after separation is a priority to ensure that the precision is unbiased as well as high.

The extension of these methods to polarization is under active development. For CBI, the polarization pipeline using generalizations of the Myers et al. (2003) techniques has been implemented. For Boomerang, the basic tools being developed include a generalization of the signal-noise separator used in Boomerang-98 (Prunet et al. 2001; Dore et al. 2002), and a variety of power spectrum estimators such as Spice (correlation function techniques (Chon et al. 2003)), Faster (approximate $\ell$ space techniques (Netterfield et al. 2002; Ruhl et al. 2003)) and Madcap (full matrix methods (Borrill 1999)). 


\section{(b) Calibrating by power spectra}

The maximum-likelihood values of the calibration and beam parameters and the errors on them that are determined in the calculation of the optimal power spectra of $\S 1 \mathrm{a}$ are themselves very useful: for the power spectra to be consistent with one derived from an underlying Gaussian model for the anisotropies, the experimentally quoted uncertainties are often reduced, as shown in table 1. Even without the excellent overall amplitude accuracy to $0.5 \%$ of WMAP, self-calibration among the pre-WMAP experiments was giving some of the same factors, which is why the grand unified spectra look so good relative to WMAP. Overall figure 2 shows an amazing concordance of data. Direct calibration using source observations or maps on equivalent regions is preferable. This has been done for Boomerang with WMAP maps by Hivon $(\approx 0.95 \pm 0.02)$ and for CBI with WMAP observations of Jupiter, the CBI calibrating source. It is remarkable how close these recalibrations are to those given here using global power spectrum analyses.

The CBI one-year data started with a \pm 0.05 calibration error. Running the optimal spectrum with WMAP, CBI is predicted to have its calibration lowered by $0.970 \pm .037$. For the two-year CBI data, we begin with the January 2003 uncertainty of \pm 0.033 and get $0.970 \pm .022$. The Jupiter calibration with WMAP resulted in a $3 \%$ downward calibration with a $1.3 \%$ calibration error (Readhead et al. 2003). The same calibration adjustment and uncertainty apply to the VSA, which also used Jupiter.

\section{Technical notes}

The WMAP power spectrum used for this calibration and beam analysis was the 49-band compression of the $\ell \leq 900$ spectrum and the optimal compression was onto the 18 bands of June 2002 in figure 2, but the results are very robust: other compressions for either WMAP or the optimal spectra, varying the $\mathcal{C}_{\ell}$ shape of figure 2 used to get the bandpowers, and adding more experiments in the calibrating mix make little difference.

\section{(c) The phenomenology of peaks and dips}

The emerging structure in the evolving power spectra of figure 2 has stimulated a number of 'model-independent' approaches to determining the statistical significance of peaks and dips. Here we apply a simple procedure we have used on Boomerang and CBI. We model the local band-power profile as the quadratic form

$$
\mathcal{C}_{\mathrm{b}}=\mathcal{C}_{p k}+\kappa_{p k}\left\langle\left(\ell-\ell_{p k}\right)^{2}\right\rangle_{\mathrm{b}} / 2
$$

where $b=1, . ., N$ runs over the number of bands $N$ we include as we slide over the data, and \langle\rangle$_{\mathrm{b}}$ denotes band-average. The position $\ell_{p k}$, amplitude $\mathcal{C}_{p k}$, and curvature $\kappa_{p k}$, are treated as 'internal' parameters, just as calibration and beam uncertainties are: they relax to their maximum-likelihood values and errors are estimated from the curvature matrix of the likelihood function about this maximum. A numerical indication of the significance of a detection is the number of sigma the peak curvature differs from zero, $\left|\kappa_{p k . m}\right| / \sigma_{\kappa_{p k}}$. To detect a peak, we require that this significance exceeds unity, and also that $\ell_{p k, m}$ lies within the range of multipoles covered by the bands. The results of applying this algorithm to the evolving grand 
unified spectra and to WMAP are shown in table 2. No attempt was made to optimize these detections by exploring band positioning and spacings.

The first peak was hinted at in the April 1999 data, was seen in the January 2000 data when Toco and Boomerang-NA were added, and localization improved by January 2002 with Boomerang, Maxima, and DASI, and then with ARCHEOPS by January 2003, and now to very high accuracy by WMAP. Boomerang+DASI (2001) detected the second and third peaks, first and second dips, and CBI (2002) detected the second, third, and fourth peaks and the third and fourth dips, albeit with some at low significance.

\section{Technical notes}

The slider bandwidth $N$ we choose depends upon the band-spacing. For the unified spectra of the evolving data, we compressed onto the $\Delta \ell=50$ bins used for Boomerang up to 900, then onto a 'CBI odd' binning used for the two-year data, although the $\mathrm{CBI}$ bands used were for the 'CBI even' binning: the odd binning is more similar to the ACBAR spacing. For the June 2002 data, CBI one-year $\Delta \ell=140$ odd bins were used. Sliders of width $N=3,4,5$ were tried, $N=3$ being more appropriate for high $\ell$ values and $N=4$ being more appropriate for lower values, to keep the the $\ell$-range probed limited to a few hundred. WMAP data were first compressed onto this Boomerang binning before the optimal spectra were constructed for the March 2003 data, but results are relatively insensitive to this and to band locations and widths. For ARCHEOPS, the $\Delta \ell$ are smaller, so larger $N$ gives the most robust detections.

Table 3 is another approach to peak/dip detection: given a class of theoretical models with a sequence of peaks and dips, the statistical distribution of positions and amplitudes can be predicted by ensemble-averaging over the full probability, the multidimensional likelihood.

\section{(d) Characteristic scales: sound crossing, peaks and dips, and damping}

A strong first peak followed by a sequence of smaller peaks diminished by damping in the $\mathcal{C}_{\ell}$ spectrum was a long-standing prediction of adiabatic models (pillar 2). The critical scale determining the spatial positions of the acoustic peaks in the spectra of figure 1 is the (comoving) sound crossing distance at recombination, $r_{s}$. The corresponding multipole scale is $\ell_{s} \equiv \mathcal{R}_{d e c} / r_{s}$, where $\mathcal{R}_{\text {dec }}$ is the angular-diameter distance that maps angles observed at our location to comoving spatial scales at recombination.

In terms of the comoving distance $\chi_{\text {dec }}$ to photon decoupling (recombination, at redshift $z_{d e c}=a_{d e c}^{-1}-1$ ), and the curvature scale $d_{k}, \mathcal{R}_{d e c}$ is given by

$$
\mathcal{R}_{\text {dec }}=\left\{d_{k} \sinh \left(\chi_{d e c} / d_{k}\right), \chi_{d e c}, d_{k} \sin \left(\chi_{d e c} / d_{k}\right)\right\},
$$

where $d_{k}=3000\left|\omega_{k}\right|^{-1 / 2} \mathrm{Mpc}$ and

$$
\chi_{d e c}=6000 \mathrm{Mpc} \int_{\sqrt{a_{d e c}}}^{1}\left(\omega_{m}+\omega_{Q} a^{-3 w_{Q}}+\omega_{k} a\right)^{-1 / 2} d \sqrt{a} .
$$

The three cases are for negative, zero and positive mean curvature. Thus the mapping depends upon $\omega_{k}, \omega_{Q}$ and $w_{Q}$ as well as on $\omega_{m}$. 
The sound crossing distance at recombination is

$$
r_{s}=\frac{6000}{\sqrt{3}} \operatorname{Mpc} \int_{0}^{\sqrt{a_{d e c}}}\left(\omega_{m}+\omega_{e r} a^{-1}\right)^{-1 / 2}\left(1+\omega_{b} a /\left(4 \omega_{\gamma} / 3\right)\right)^{-1 / 2} d \sqrt{a},
$$

where $\omega_{\gamma}=2.46 \times 10^{-5}$ is the photon density and $\omega_{e r}=1.68 \omega_{\gamma}$ for three species of massless neutrinos.

The estimates of $r_{s}$ and $\pi \ell_{s}$ have been quite stable over time (table 3). Values are determined by averaging over the $\mathcal{C}_{\ell}$ model space probabilities. Since the $r_{s}$ are comoving, the physical sound horizon at decoupling is ca. $140 \mathrm{kpc}$.

The angular-diameter-distance relation maps spatial structure at photon decoupling perpendicular to the line-of-sight with transverse wavenumber $k_{\perp}$ to angular structure, through $\ell=\mathcal{R}_{d e c} k_{\perp}$. Converting peaks in $k$-space into peaks in $\ell$-space is complicated by three-dimensional to $2 \mathrm{D}$ projection effects over the finite width of decoupling and also by the influence of sources other than sound oscillations such as Doppler terms.

The peak locations $\ell_{p k, j}$ in table 3 are obtained by forming $\exp <\ln \ell_{p k, j}>$, where the average and variance of $\ln \ell_{p k, j}$ are determined by integrating over the probability-weighted $\mathcal{C}_{\ell}$ database. These peak locations accord reasonably well with $\ell_{p k, j} \approx j f_{j} \pi \ell_{s}$, where the numerically estimated constant $f_{j} \approx 0.75$ for the first peak, approaching unity for higher ones. (There are small $n_{s}-1$ corrections to $\ln f_{j}$.) The interleaving dips are also shown. Dip locations are well determined by replacing $j$ by $j+1 / 2$, with slightly different $f_{j}$ factors.

For fixed $\omega_{b}$ and $\omega_{m}$, constant $\ell_{s}$ lines in the $\Omega_{k}-\Omega_{\Lambda}$ and $w_{Q}-\Omega_{Q}$ planes look rather similar to contour lines determined from the data (see figures 5 and 7 in Bond et al. 2003a). This degeneracy (Efstathiou \& Bond 1999) among these parameters would be exact except for the integrated Sachs-Wolfe effect. However, lines of constant $H_{0}$ are different in that space and break the degeneracy, only weakly for the weak prior but more so when the HST-h or the SN1 prior on deceleration is imposed. The degeneracy in the $\Omega_{k}-\Omega_{\Lambda}$ plane is also broken when LSS information is added, though less so in the $w_{Q}-\Omega_{Q}$ plane.

Also evident in the spectra in figure 1 and the diminishment of the peak heights in table 3 is the damping tail, an overall decline due to the shear viscosity and the finite width of the region over which hydrogen recombination occurs. Scales for both can be estimated analytically and are similar. Values for the comoving scale $R_{D}$ and an associated angular damping scale $\ell_{D} \equiv \mathcal{R}_{\text {dec }} / R_{D}$ are given in table 3 . The physical scale $R_{D} /\left(1+z_{\text {dec }}\right)$ at recombination is therefore about 10 kpc. (The decline in $\mathcal{C}_{\ell}$ is parameterized by a $\exp \left[-\left(\ell / \ell_{D}\right)^{m_{D}}\right]$ multiplier (Hu and White 1996). We find $m_{D}=1.27_{-0.008}^{+0.008}$ for January 2003 and $1.27_{-0.003}^{+0.003}$ for March 2003.)

The $\omega_{b}$ dependence in $r_{s}$ would lead to a degeneracy with other parameters in terms of peak/dip positions. However, relative peak/dip heights are extremely significant for parameter estimation as well, and this breaks the degeneracy. For example, increasing $\omega_{b}$ beyond the nucleosynthesis (and CMB) estimate leads to a diminished height for the second peak that is not in accord with the data. Page et al. (2003) used the peak/dip parameters to estimate directly the cosmic parameters. 


\section{Cosmic parameter estimations}

\section{(a) Evolution of marginalized cosmic parameters}

Table 4 and figure 4 show the evolution of cosmic parameter estimations from projected 1D likelihood curves, using the $\mathcal{C}_{\ell}$ database used in Sievers et al. (2003), Ruhl et al. (2003) and Goldstein et al. (2003), an extension of that used in Lange et al. (2001), Jaffe et al. (2001) and Netterfield et al. (2002) to reflect the growing precision. The ranges quoted are Bayesian $50 \%$ values and the errors are $1 \sigma$, obtained after projecting (marginalizing) over all other parameters. For derived variables such as $h$ amd age, ensemble-averaged means and variances are used. The WMAP addition punches out the detection in a remarkable way.

The MCMC parameter estimates for weak and weak+flat priors in table 5 agree well with the entries in table 4: about as good as one might expect given the differences. In particular, WMAP with MCMC includes the $\tau_{C}$ constraint by explicit calculation of TE likelihoods, while the database either lets the TT data alone decide or uses the broader-than-Gaussian $\tau_{C}$ prior to reflect the detection. The March 2003 results in the table include this prior, but most parameters are insensitive to it: even $\sigma_{8}$ does not migrate that much.

The Spergel et al. (2003) MCMC four-chain results for flat power law $\Lambda$ CDM models for WMAP-only are quite similar to what we obtain. Their WMAP-ext consists of WMAP plus cut verions of the ACBAR and CBI one-year power spectra. Our March 2003 set contains more data and allows more overlap. Their analogue of LSS is to use the 2dFRS power spectrum. This assumes a linear scale-independent bias. It is a more stringent prior on shape than our $\Gamma$ constraint. They also add (small-scale) Ly $\alpha$ forest information to extend the $k$-space coverage. The forest data could be sensitive to gastrophysical complications, and we would hesitate to construct any but a very weak prior for it at this time.

We also find good agreement with Contaldi, Hoekstra and Lewis (2003), who applied Cosmomc to the WMAP+ACBAR+CBI data and the Red Cluster Survey (RCS) weak-lensing data. Our LSS prior was designed to encompass $\sigma_{8}$ constraints from weak lensing displayed in figure 5 , and RCS is one of those entries.

Table 4 shows that with just the weak prior, there are strong detections for $\Omega_{t o t}$, $\omega_{b}, \omega_{c d m}$ and $n_{s}$. The HST-h prior helps to determine $\Omega_{\Lambda}$ better than the weak prior, because it breaks more strongly the $\Omega_{k}-\Omega_{\Lambda}$ near-degeneracy. SN1 breaks it even more strongly, and the HST-h, weak+SN1 and weak+LSS results are all compatible, showing there is no very strong tension among these priors.

The precision of WMAP invites exploration of larger parameter spaces. A first issue is whether $n_{s}$ varies. When Spergel et al. (2003) added the high- $\ell$ ACBAR and CBI data to make their WMAP-ext dataset, they found lower $n_{s}$, and further adding the 2dFRS data and the small-scale data from the Lyman alpha forest exacerbated the issue. We find the same result with the database with MCMC: $n_{s}$ changes by about 0.03 from WMAP-only to the March 2003 set for the weak+flat prior, and by a further 0.015 with LSS added.

Modelling $n_{s}$ variation with a logarithmic correction, Spergel et al. (2003) get $n_{s}$ varying from 1.2 to 0.93 . (See also Bridle et al. 2003, who show that dropping low- $\ell$ modes reduces the significance of running index detection.) The lower panel of table 5 shows how the parameters change relative to the fixed $n_{s}$ model when this extra parameter is introduced, for the weak+flat prior and for the various datasets. We 
restricted $d n_{s} / d \ln k$ to lie within the range $-0.2-0.2$. The normalization point for $n_{s}$ is $k_{n}^{-1}=20 \mathrm{~h}^{-1} \mathrm{Mpc}$, and the horizon scale is $\chi_{\text {dec }} \approx 0.88\left(6000 / \Omega_{m}^{1 / 2}\right) \mathrm{h}^{-1} \mathrm{Mpc}$ for a $\Lambda$ CDM model, so $\ln k_{n} \chi_{d e c} \sim 6$, hence substantial slope changes are possible for the $d n_{s} / d \ln k$ values obtained. Although table 5 shows there is a preference for a logarithmically varying slope from January 2002, due to downturns in the CMB data at low and high $\ell$, it is $\lesssim 2 \sigma$ in likelihood fall-off even in the March 2003 data. The likelihood maxima are also smaller than the means given in the table. We also caution that $d n_{s} / d \ln k\left(k_{n}\right)$ is correlated with the $\tau_{C^{-}} \sigma_{8}-n_{s}$ combination, which results in upward extension of the $1 \mathrm{D}$ distributions for $\tau_{C}$ and $\sigma_{8}$. Prior choices on $\tau_{C}$ can then move the limits around: the weak+flat results in table 5 place no restriction beyond the weak 0.7 upper boundary on $\tau_{C}$ to conform MCMC to our database limit.

The WMAP team estimate the gravitational wave (tensor) contribution to be $<0.72$ of the scalar component in amplitude.

We have considered variations in the dark energy equation of state. For the database calculations, we used a flat prior and allowed $w_{Q}$ to vary from -1 to -0.01 , as in Bond et al. (2000). We applied it to the June 2002 data (and obtained $w_{Q}<-0.7$ at the $95 \% \mathrm{CL}$ for the prior combination weak+flat+LSS+SN1) (Bond et al. 2003a) and to what was almost the same as the January 2003 data (for which $w_{Q}<-0.8$ was obtained) (Pogosyan et al. 2003). MCMC calculations yield about the same limits: less than -0.70 at $2 \sigma$ for the HST-h+flat+LSS and SN1 prior. We get only slightly better limits for the March 2003 data (less than -0.71). This constraint is SN1-driven (for weak $+\mathrm{SN} 1 w_{Q}<-0.68$ ) rather than HST-h-driven $\left(w_{Q}<-0.48\right)$. Although bands of allowed $h$ do break the angular-diameter-distance degeneracy between $\Omega_{Q}$ and $w_{Q}$, allowed bands for the deceleration parameter $q_{0}$ break it more effectively.

Unlike the WMAP team, our weak+flat+LSS prior does not give a good constraint. Since the shape parameter $\Gamma \sim \omega_{m} / h$, and $\omega_{m}$ is now accurately determined by the CMB data, our shape constraint approaches a pure $h$ prior. For $\omega_{m} \sim 0.14$, it is similar to the weak limits we are already imposing on $h$. The 2 dFRS data give $\Gamma=0.21 \pm 0.03$ and the SDSS data give $0.19 \pm 0.04$, so the combination would nomimally have an $11 \%$ relative Gaussian error, the same as for the HST-h prior, suggesting the constraint would be similar if this strong-LSS were to be imposed.

Why the limits are typically $<-0.7$ or -0.8 with SN1 is easy to understand: the $2 \sigma$ SN1 error contour in the $\Omega_{Q}-w_{Q}$ plane roughly follows a $q_{0} \approx-1 / 4$ line, where $q_{0}=\left(1+3 w_{Q} \Omega_{Q}\right) / 2$ is the current deceleration parameter. With $\Omega_{Q} \gtrsim 0.65$, this gives the -0.7 to -0.8 range for the limit. The way the CMB comes into this is to restrict the allowed values of $\Omega_{Q}$, and it used to be that the data was such that the LSS prior was needed to do this the first time we went through this exercise. The addition of WMAP refines $\Omega_{Q}$ but this only fine tunes the limit by a small amount.

The other component to our LSS prior, the amplitude constraint $\sigma_{8}$, has had a significant impact in constraining models. The June 2002 data for the weak + flat prior give $\sigma_{8}=0.88 \pm 0.11$ and when LSS is added, $0.86 \pm 0.08$ (Bond et al. 2003b). For the March 2003 data, the MCMC calculation with the weak+flat prior gives $0.85 \pm 0.06$, the database calculation with the weak + flat $+\tau_{C}$ prior gives $0.83 \pm 0.06$ using the 899-point WMAP data and $0.81 \pm 0.06$ using the compressed 98-band WMAP data. Adding the LSS prior gives $0.86 \pm 0.05,0.85 \pm 0.04$ and $0.86 \pm$ 
0.04 , respectively. (These $\sigma_{8}$ differences, plus $\sim 0.01$ excursions in $n_{s}$, represent the biggest deviations between compressed and uncompressed WMAP estimations; and the up and down $1 \sigma$ and $2 \sigma$ limits are close.)

The SZ effect breaks the $\sigma_{8}-\tau_{C}$ degeneracy. The SZ power spectrum is found to scale as $\sigma_{8}^{7}$ about a $\Lambda$ CDM model with $\sigma_{8}=0.9$ (e.g. Bond et al. 2003b). This high non-linearity means that it could be an excellent way to estimate $\sigma_{8}$. For an $\mathrm{SZ}$ explanation to work for the (recalibrated) CBI+BIMA+ACBAR data seems to require $\sigma_{8} \approx 0.94_{-0.16}^{+0.08}$ with $1 \sigma$ errors which include the non-Gaussian nature of the SZ effect in the small patches that the CBI deep and BIMA measurements probe (Goldstein et al. 2003). These SZ distributions overlap with those found for the CMB and CMB+LSS data, but the jury is still out on whether this is the explanation.

The calculation of the SZ angular power spectrum requires detailed hydrodynamical simulations that properly take into account cluster structure, pressure profiles, heating/cooling, etc. There are still uncertainties as to the role of feedback, and much work is needed on the theoretical side as well as the observational side to turn this technique into a high-precision tool.

\section{(b) Parameter eigenmodes and degeneracy breaking}

To help break parameter near-degeneracies via CMB alone, one can decrease $\Delta \ell$ of the bands with fixed error, as ARCHEOPS and WMAP did. We can also extend the $\ell$ range, which $\mathrm{CBI}$, ACBAR and the VSA have done. Planck will do both.

Polarization helps, by breaking the $\tau_{C}$-amplitude near-degeneracy and also by breaking the $n_{s}(k)$ degeneracy that arises if we let the index have full functional freedom. The EE power near the peak will be well probed by many of the polarization experiments mentioned in $\S 2 \mathrm{f}$.

Degeneracy breaking also arises when non-CMB data are added, as the HST-h, SN1a, and LSS examples show. Higher order statistics can also help, e.g. skewness/kurtosis.

Forecasting how proposed experiments would improve on cosmic parameter errors became, and remains, quite a cottage industry. As mentioned in $\S 1$ e, a figure of merit is how many parameter eigenmodes will be determined to a specific precision level, i.e. the number of modes with $\sigma_{\alpha}$ below some number, taken here to be 0.1 and 0.01 , respectively.

How well did we do? The forecast for Boomerang+DMR for $1.8 \%$ of the sky with four $150 \mathrm{GHz}$ bolometers gave four out of nine linear combinations should be determined to \pm 0.1 accuracy. This is what was obtained in the full analysis of Netterfield et al. (2002). (For the forecasts, $\omega_{h d m}$ and $n_{t}$ were included in the parameter mix, $w_{Q}$ was frozen at -1 , making nine.) The forecast for WMAP with two years of data was $6 / 9$ to \pm 0.1 and $3 / 9$ to \pm 0.01 . For one year of WMAP-only data and the weak prior, $2 / 7$ are determined to $\pm 0.01,5 / 7$ to \pm 0.1 ; adding the $\tau_{C}$ prior increase this to $6 / 7$. (For Planck, $5 / 9$ to \pm 0.01 accuracy are predicted.)

The March 2003 eigenmodes for the weak prior are: to \pm 0.004 , a primarily $\Omega_{k^{-}}$ $\Omega_{\Lambda}$ combination, related to $\pi \ell_{s}$; to \pm 0.008 , a $\ln \mathcal{C}_{10}-\tau_{C}-n_{s}$ combination; to \pm 0.02 , $\omega_{b}$ predominantly, with some extra; to \pm 0.04 a $\ln \mathcal{C}_{10}-\tau_{C}-n_{s}-\omega_{c d m}$ combination, and another to \pm 0.08 . The sixth, \pm 0.13 , is in a direction that would break the amplitude $\tau_{C}$ degeneracy. When the $\tau_{C}$ prior is included, this error diminishes. The least well 
determined mode is associated with the $\Omega_{k}-\Omega_{\Lambda}$ angular-diameter-distance degeneracy. Goldstein et al. (2003) give the eigenmodes for what is basically the January 2003 mix except the June 2002 CBI and VSA data are used. The January 2003 data used here have best determined modes, to $\pm 0.011, \pm 0.015, \pm 0.05$, that are mainly similar to the March 2003 ones, but with differing subdominant structure in the linear combinations.

Whether before or after WMAP, the simplest inflationary paradigm with minimal parameters fits the data well. This does not mean inflation is proved, but competitor theories would have to look awfully like inflation for them to work. As CMB precision increases at high $\ell$, and more polarization data arrive, and as the LSS and SN1 data improve, more details with more parameters will be explored. Already WMAP points once again to the low- $\ell$ anomaly and with the other data to possible variation in the primordial slope with wavenumber.

We thank our collaborators on the Boomerang, CBI and ACBAR teams for sharing the exhilaration of spectral revelation over the past few years and Antony Lewis for Cosmomc and other discussions. Lastly we would have you picture a beaming Dave Wilkinson with Sunyaev and Bond in front of a Saturn V rocket of the sort that took the US to the Moon, taken shortly before the Delta-launch of WMAP in June 2001, and the celebration thereafter - still ongoing, but greatly missing the $\mathrm{W}$ in WMAP.

\section{References}

Bennett, C. L. et al. 2003 Astrophys. J. Supp. 148, 1, astro-ph/0302207. WMAP

Benoit, A. et al. 2003 Astron. Astrophys. 399, L19, L25. ARCHEOPS

Bond, J.R. 1996 Cosmology and large scale structure. Les Houches Session LX (ed. R. Schaeffer J. Silk, M. Spiro \& J. Zinn-Justin), pp. 469-674. Elsevier. (Available at http://www.cita.utoronto.ca/ bond/papers/houches/LesHouches96.ps.gz)

Bond, J.R. \& Crittenden, R.G. 2001 in Proc. NATO ASI, Structure Formation in the Universe, (es. R.G. Crittenden \& N.G. Turok) Dordrecht:Kluwer. astro-ph/0108204

Bond, J.R., \& Jaffe, A. 1999 Phil. Trans. R. Soc. Lond. A 357, 57. astro-ph/9809043

Bond, J.R., Jaffe, A.H., \& Knox, L. 1998 Phys. Rev. D 57, 2117.

Bond, J.R., Jaffe, A.H. \& Knox, L. 2000a Astrophys. J. 533, 19.

Bond, J.R., Pogosyan, D. \& Souradeep, T. 2000b Phys. Rev. D 62, 043006. astro$\mathrm{ph} / 9912144$

Bond, J.R. et al. 2000c In 'Cosmology 65 Particle Physics, Proc. CAPP 2000 (ed. J. GarciaBellido, R. Durrer, M. Shaposhnikov), Washington, DC: American Institute of Physics. astro-ph/0011379

Bond, J.R. et al. 2003a In MRST 2002: a tribute to George Leibbrandt (ed. R. Epp, V. Elias, R. Myers). AIP Conference Proceedings Series, vol. 646, pp. 15-33. Bethelehem, PA: American Institute of Physics. astro-ph/0210007

Bond, J.R. et al. 2003b Astrophys. J. in press. astro-ph/0205386

Borrill, J. 1999 Phys. Rev. D 59, 027302 (http://www.nersc.gov/ borrill/cmb/madcap/)

Bridle, S.L., Lewis, A.M., Weller, J. \& Efstathiou, G. 2003 Mon. Not. R. Astr. Soc. 342, L72.

Chon, G., Challinor, A., Prunet, S., Hivon, E. \& Szapudi, I. 2003 Mon. Not. R. Astr. Soc. in press. astro-ph/0303414, Spice

Christensen, N. \& Meyer, R. 2001 Phys. Rev. D 64, 22001.

Christensen, N., Meyer, R., Knox, L. \& Luey, B. 2001 Class. Quant. Grav. 18, 2677.

Chu, M., Kaplinghat, M. \& Knox, L. 2003 Astrophys. J. in press. astro-ph/0212466

Phil. Trans. R. Soc. Lond. A 361, 2435 (2003) 
Contaldi, C., Hoekstra, H. \& Lewis, A. 2003 Phys. Rev. Lett. 90, 221303. astro-ph/0302435

Dawson, K.S., Holzapfel, W.L., Carlstrom, J.E., LaRoque, S.J., Miller, A., Nagai, D. \& Joy, M. 2002 Astrophys. J. 581, 86-95. astro-ph/0206012, BIMA

de Bernardis, P. et al. 2000 Nature 404, 995. Boom00

de Bernardis, P. et al. 2002 Astrophys. J. 564, 559. Boom01

Dore, O., Teyssier, R. Bouchet, F.R., Vibert, D. \& Prunet, S. 2002 Astron. Astrophys. in press. astro-ph/0101112 Mapcumba

Efstathiou, G. \& Bond, J.R. 1999 Mon. Not. R. Astr. Soc. 304, 75.

Freedman, W. et al. 2001 Astrophys. J. 553, 47

Goldstein J. et al. 2003 Astrophys. J. in press. aph/0212517, ACBAR

Grainge, K. et al. 2003 Mon. Not. R. Astr. Soc. in press. astro-ph/0212495, VSA-ext

Halverson, N.W. et al. 2002 Astrophys. J. 568, 38. DASI

Hanany, S. et al. 2000 Aatrophys. J. Lett. 545, 5. Maxima00

Hinshaw, G. et al. 2003 Astrophys. J. Supp. 148, 135, astro-ph/0302217, WMAP TT

Hivon, E., Gorski, K.M., Netterfield, C.B., Crill, B.P., Prunet, S. \& Hansen, F. 2002 Astrophys. J. in press. astro-ph/0105302, Master

Hivon, E. \& Kamionkowksi, M. 2002 Science 298, 1349. astro-ph/0211553

Hu, W. \& White, M. 1996 Astrophys. J. 471, 30.

Jaffe, A.H. et al. 2001 Phys. Rev. Lett. 86, 3475.

Kirkman, D., Tytler, D., Suzuki, N., O' Meara, J. \& Lubin, D. 2003 Astrophys. J. Supp. in press. astro-ph/0302006, bbn

Kogut, A. et al. 2003 Astrophys. J. Supp. 148, 161. astro-ph/0302213, WMAP TE

Kosowsky, A., Milosavljevic, M. \& Jiminez, R. 2002 Phys. Rev. D 66, 063007.

Kovac, J. et al. 2002 Nature 420, 772-787. astro-ph/0209478, DASIpol

Kuo, C.L. et al. 2003 Astrophys. J. in press. aph/0212289, ACBAR

Lange, A. et al. 2001 Phys. Rev. D 63, 042001. Boom00

Lee, A.T. et al. 2002 Phys. Rev. D 561, L1. Maxima01

Leitch, E.M. et al. 2003 Nature 420, 763-771. astro-ph/0209476, DASIpol

Lewis, A. and Bridle, S. 2002 Phys. Rev. D 66 20103511. (And references therein, available at http://cosmologist.info/cosmomc).

Lewis, A., Challinor, A., \& Lasenby, A. 2000 Astrophys. J. 538, 473. (http://camb.info/)

Mason, B. et al. 2003 Astrophys. J. 591, 540. astro-ph/0205384, CBI deep

Mauskopf, P. et al. 2000 Aatrophys. J. Lett. 536, L59. Boom-NA

Metropolis, N., Rosenbluth, A.W., Rosenbluth, M.N., Teller, A.H, \& Teller, E. 1953 J. Chem. Phys. 21, 1087.

Miller, A.D. et al. 1999 Astrophys. J. 524, L1. Toco

Myers, S.T. et al. 2003 Astrophys. J. 591, 575. astro-ph/0205385, CBI

Netterfield, B. et al. 2002 Astrophys. J. 571, 604. Boom01

Page, L. et al. 2003 Astrophys. J. Supp. 148, astro-ph/0302220, WMAP

Pearson, T.J. et al. 2003 Astrophys. J. 591, 540. astro-ph/0205388, CBI mosaic

Perlmutter, S. et al. 1999a Astrophys. J. 517, 565. SN1

Perlmutter, S., Turner, M. \& White, M. 1999b Phys. Rev. Lett. 83, 670. SN1

Perlmutter, S. \& Schmidt, B.P. 2003, astro-ph/0303428. SN1

Pogosyan, D., Bond, J.R., \& Contaldi, C. 2003 In Proc. XVIII IAP Colloq. On the Nature of Dark Energy, Paris, 1-5 July 2002. astro-ph0301310

Prunet, S. et al. 2001 In Mining the Sky, Proc. MPA/ESO/MPA Conf., Garching, Germany, 31 July-4 August 2000. astro-ph/0101073

Readhead, A.C. et al. 2003 preprint, CBI two-year

Riess, A. et al. 1998 Astron J. 116, 1009. SN1 
Ruhl, J.E. et al. 2003 Astrophys. J. in press. astro-ph/0212229, Boom02

Seljak, U. \& Zaldarriaga, M. 1996 Astrophys. J. 469, 437. CMBfast

Scott, P.F. et al. 2002 Mon. Not. R. Astr. Soc. 341, 1076. astro-ph/0205380, VSA

Sievers, J. et al. 2003 Astrophys. J. 591, 599. astro-ph/0205387, CBI

Spergel, D. N. et al. 2003 Astrophys. J. Supp. 148, 175. astro-ph/0302209, WMAP

Verde, L. et al. 2003 Astrophys. J. Supp. 148, 195. astro-ph/0302218, WMAP

Phil. Trans. R. Soc. Lond. A 361, 2435 (2003) 


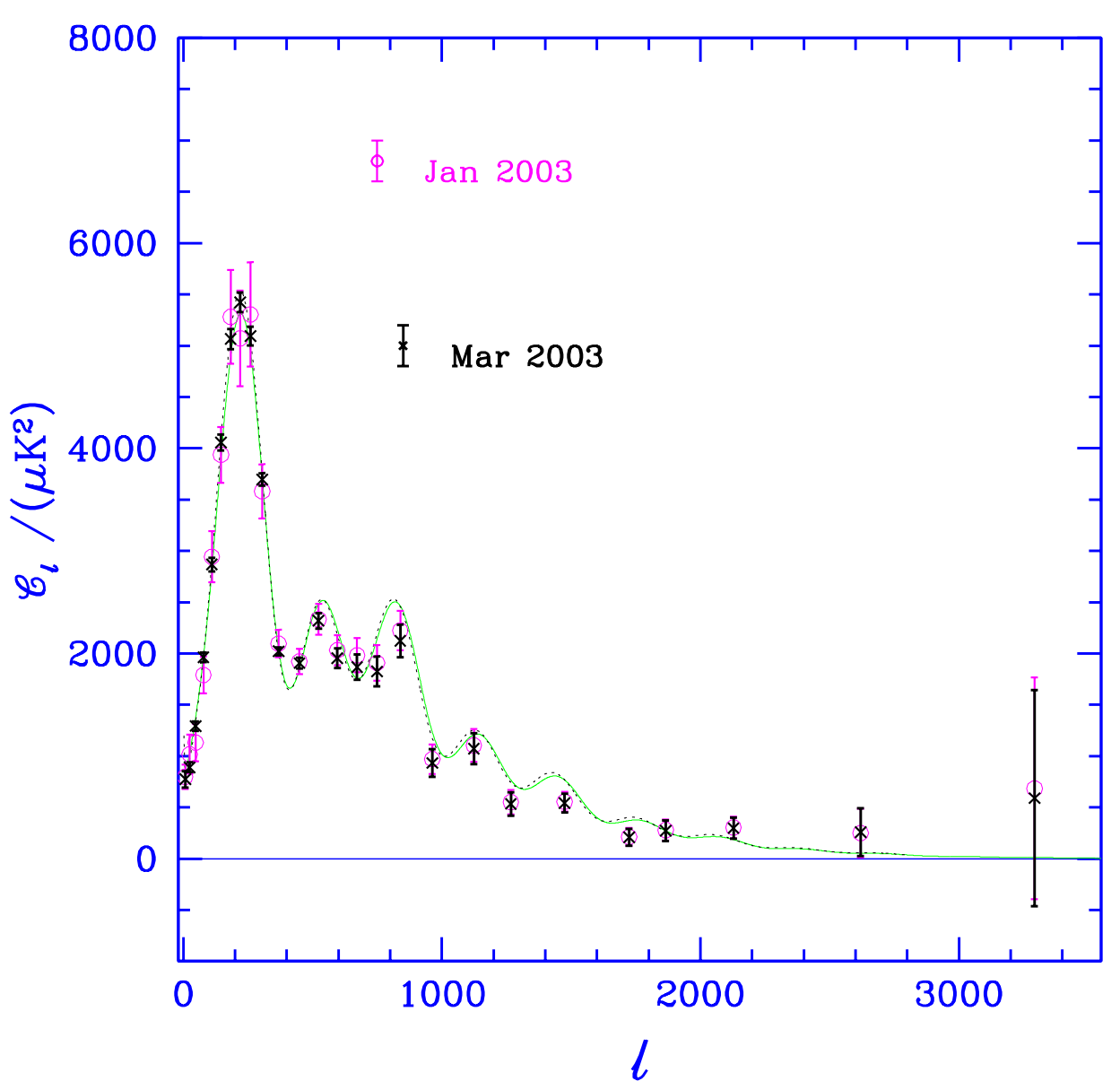

Figure 1. Optimal $\mathcal{C}_{\ell}$ spectra for the pre-WMAP January 2003 data and the post-WMAP March 2003 data show good agreement. These spectra are maximum-likelihood determinations of the power in 26 (top-hat) bands, with calibration and beam uncertainties of the various experiments fully taken into account. Two $\mathcal{C}_{\ell} \Lambda$ CDM models from the database are shown. The dotted (black) curve best fits the March 2003 data with the weak + flat $+\mathrm{LSS}+\tau_{C}$ prior applied. It has parameters $\left\{\Omega_{\mathrm{tot}}, \Omega_{\Lambda}, \Omega_{b} h^{2}, \Omega_{\mathrm{cdm}} h^{2}, n_{s}\right.$, $\left.\tau_{C}, t_{0}, h, \sigma_{8}\right\}=\{1.0,0.7,0.0225,0.12,0.975,0.15,13.7,0.69,0.89\}$. The solid green curve that looks quite similar best fits the June 2002 data for the weak+flat+LSS prior (Sievers et al. 2003), with parameters $\{1.0,0.5,0.020,0.14,0.925,0,14.4,0.57,0.82\}$. It was used as the inter-band shape for this optimal bandpower determination, but the results are insensitive to this. The bandpowers are optimally placed in $\ell$. Their finite horizontal extension is not shown, and the vertical diagonal bandpower errors also do not show the whole story since there are band-to-band correlations. (e.g. the visual up-down-up at the first peak for January 2003 is indicative of the strong correlations and can disappear with different banding, e.g. one better tuned to Boomerang's binning.) Despite these caveats, the best fit $\mathcal{C}_{\ell}$ would fit better with a slight downward tilt beyond $\ell \gtrsim 500$, which a scale-dependent $n_{s}(k)$ could do (see $\left.\S 4\right)$. 


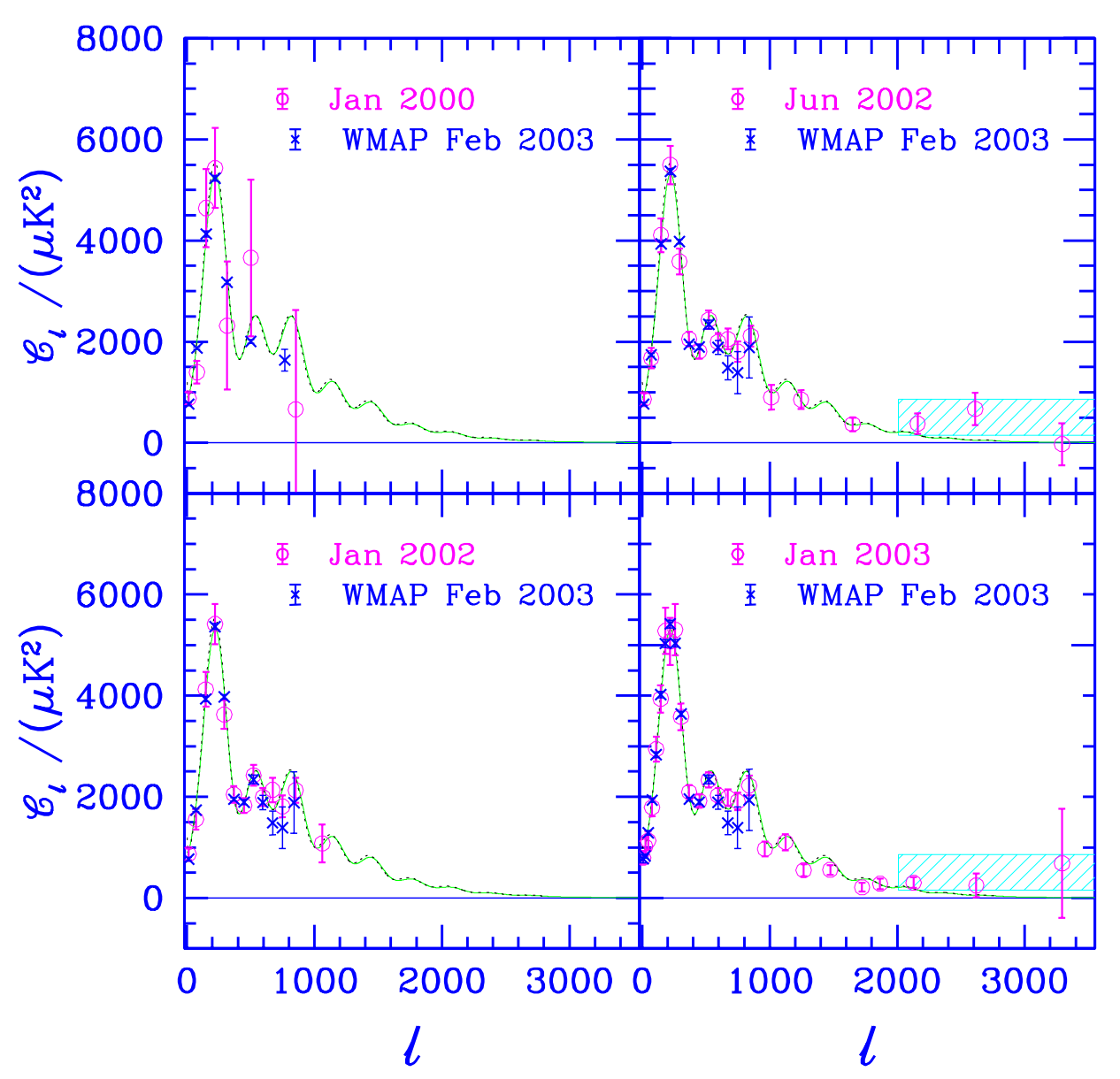

Figure 2. The evolving optimal $\mathcal{C}_{\ell}$ values are compared with WMAP-only power spectra (crosses) compressed onto the same bands. The degree of compression does not do visual justice to WMAP because the errors are so small until beyond the second peak. The bands were chosen to be natural for the data at the time, but band-to-band correlations do exist. The spectra include the following data: January 2000 has DMR + Toco + Boomerang-NA + the April 1999 mix; January 2002 has January 2000 and the Boomerang data of Netterfield et al. (2001) + Maxima + DASI; June 2002 has January 2002 plus one-year CBI mosaic and deep field data and the (non-extended) VSA data; January 2003 has ARCHEOPS + ACBAR, uses the Ruhl et al. (2003) Boomerang spectrum covering $2.9 \%$ of the sky, the extended-VSA data and the two-year combined CBI mosaic + deep field data. The $\ell>2000$ excess found with the one-year deep CBI data is denoted by the light blue hatched region ( $95 \%$ confidence limit) in the right hand panels. The two best fit $\Lambda \mathrm{CDM}$ models of figure 1 are repeated in each of the panels. When HST-h or SN1 priors are included in the June 2002 data, the best fit model has the same parameters as those of the March 2003 curve, except for a slight shift in tilt, to $n_{s}=1.0$, a corresponding rise in $\tau_{C}$, to 0.20 , leading to $\sigma_{8}=0.91$. 


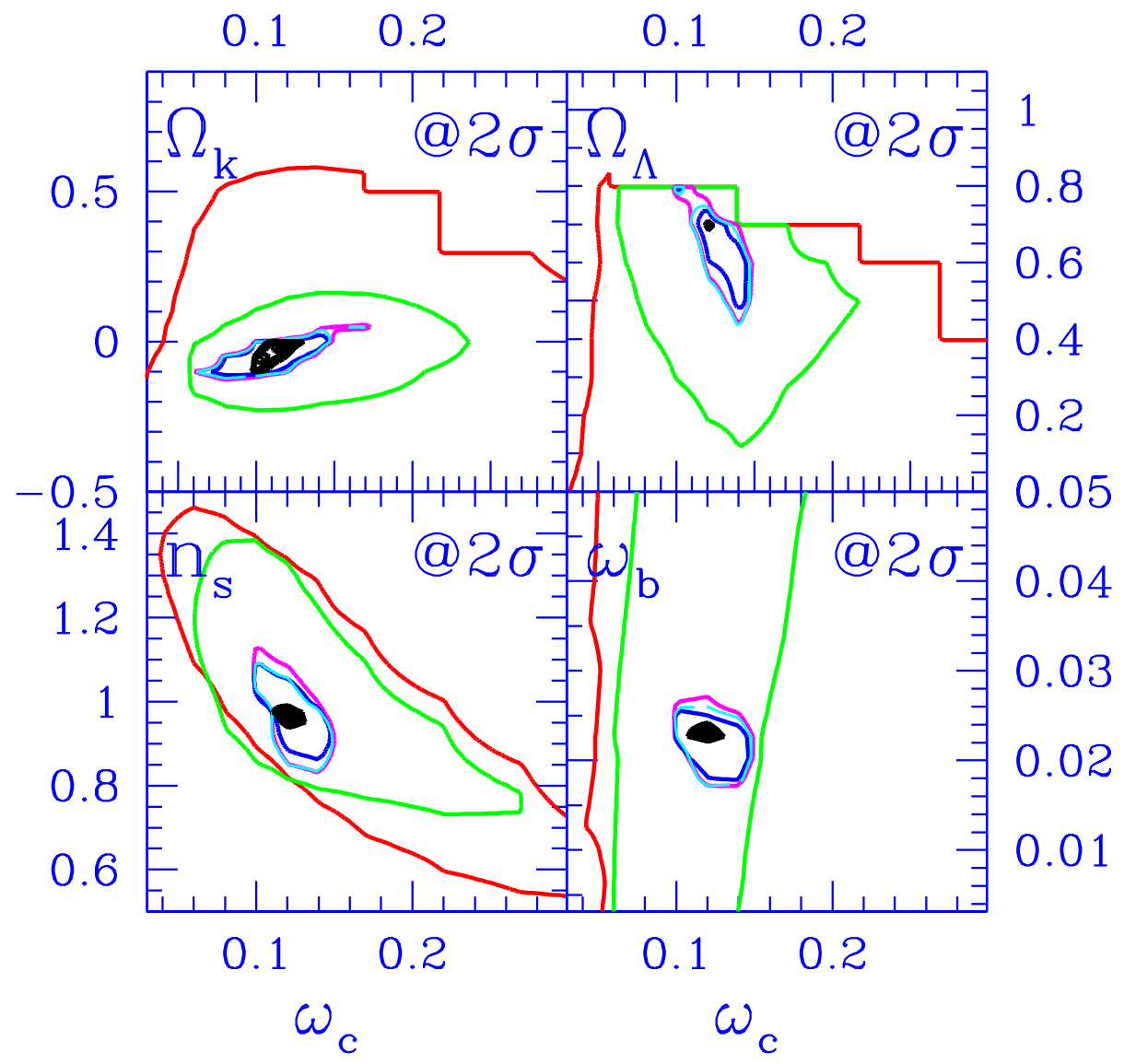

Figure 3. The evolution of $2 \sigma$ likelihood-contour regions, with the weak+LSS prior probability applied in the top-left-hand panel, and the flat $\Omega_{t o t}=1$ applied additionally in the rest. The outer (red) contour is for COBE-DMR only, then the sequence of increasing concentration is for the January 2000 (green), January 2002 (magenta), June 2002 (cyan) and January 2003 (blue) data. The dense (black) region is for March 2003, including WMAP, but excluding ARCHEOPS and DMR because of their substantial overlap with WMAP. A $\tau_{C}$ prior (see $\S 1 \mathrm{~d}$ ) taking into account the WMAP 'model independent' TE analysis, has been included, but it does not make much difference to these results. If only the weak prior is imposed, the January 2000 data still show rough $\Omega_{k}$ localization, but do not constrain the other parameters significantly, whereas the January 2002 and January 2003 contour regions without LSS are only slightly bigger than those shown here. For March 2003, the black regions remain small, but the $\Omega_{\Lambda}-\Omega_{k}$ degeneracy becomes more evident, with closed universes and smaller $\Omega_{\Lambda}$ allowed. 
Table 1. Calibration parameters that the temperature anisotropies should be multiplied by, as determined in the computation of optimal spectra. (Before WMAP, calibrations by this method were made relative to each other, with the interferometry data (INT) dominating because of smaller uncertainties: $4 \%$ for DASI; $5 \%$ for CBI in the June 2002 data, 3.3\% in January 2003, and 1.3\% in March 2003 (Readhead et al. 2003); 3.5\% for VSA for June 2002 and January 2003, and $1.3 \%$ in March 2003. WMAP gives $0.5 \%$. INTc denotes the recalibrated CBI and VSA, along with DASI. The Boomerang calibrations shown are for the $2.9 \%$ sky-cut Ruhl et al. (2003) 'Faster' power spectrum, in conjunction with INT and/or WMAP. The values are quite robust as other experiments are added. (Similar values are obtained if we use the $1.8 \%$ Netterfield et al. (2002) cut rather than the $2.9 \%$ : $0.953 \pm 0.037,0.938 \pm 0.036,0.949 \pm 0.021$ and $0.942 \pm 0.020$ for INT, INTc, WMAP and WMAP+INTc.) ACBAR was done with Boomerang included and the 2-14 bins. (The multipliers are lower when the first bin is included, $0.94 \pm 0.05,0.92 \pm 0.05,0.89 \pm 0.047$, $0.914 \pm 0.042$.) ARCHEOPS was also done with Boomerang included for INT and INTc. The results are the same whether Boomerang is recalibrated or not. Beam adjustments are also computed by the method. For example, the Boomerang beam is compatible with no change, but substantially reduced errors: the prior is $1.00 \pm 0.13$ and we determine $1.02 \pm 0.03$ with INT, $0.984 \pm 0.026$ with WMAP and $1.00 \pm 0.022$ with WMAP+INTc. When cosmological parameters are determined, adjustments such as these are done for each experiment to maximize the entropy of each model in turn, but the uncertainties are then marginalized. The optimal bandpowers also have the calibrations marginalized.)

\begin{tabular}{|l|l|l|l|}
\hline Calib & Boom & ACBAR & ARCHEOPS \\
\hline prior & $1.00 \pm 0.10$ & $1.00 \pm 0.1$ & $1.00 \pm 0.07$ \\
INT & $0.975 \pm 0.035$ & $0.96 \pm 0.05$ & $1.06 \pm 0.034$ \\
INTc & $0.961 \pm 0.036$ & $0.947 \pm 0.044$ & $1.05 \pm 0.039$ \\
WMAP & $0.963 \pm 0.017$ & $0.912 \pm 0.049$ & $1.046 \pm 0.019$ \\
WMAP+INTc & $0.959 \pm 0.017$ & $0.938 \pm 0.044$ & $1.046 \pm 0.019$ \\
\hline
\end{tabular}

Phil. Trans. R. Soc. Lond. A 361, 2435 (2003) 
Table 2. Peak/dip locations $\ell_{p k / d i p, j}$ and heights $\mathcal{C}_{p k / d i p, j}$ (in $\mu K^{2}$ ) from maximum-likelihood analysis of data within $N$ bands as the bandwidth slides over the data. (Results for a four-band-width slider acting on the compressed grand unified spectra of the various epochs are shown. Values of the curvature $\left|\kappa_{p k / \text { dip,m }}\right|$ in units of $\sigma_{\kappa_{p k}}$ are given beside the $\ell_{p k / d i p, j}$. A detection requires that this exceeds unity and $\ell_{p k / d i p, j}$ lies within the bands probed. If a peak/dip detected with a three-band slider is more significant than for the four-band slider, it is shown in brackets. When we do WMAP-only with this crude Boomerang binning we get the results in the second column that are in excellent accord with the Gaussian-fit results of Page et al. (2003): (220 $\pm 0.8,5373 \pm 337)$, $(546 \pm 10,2381 \pm 83)$ and $(411.7 \pm 3.5,1707 \pm 43)$ for the first and second peak and the first dip. Other individual experiments we have applied this procedure to include Boomerang, CBI and ARCHEOPS. The numbers in the table also accord well with those we have obtained for Boomerang (de Bernardis et al. 2002; Ruhl et al. 2003) and CBI (Pearson et al. 2003; Readhead et al. 2003) data alone; e.g. for Boomerang-only with the Faster power spectrum, recalibrated as in table 1 , we get $(221 \pm 7,3.5,5371 \pm 337),(528 \pm 15$, $1.5,2183 \pm 136),(820 \pm 15,1.5,2000 \pm 215)$ for peaks and $(412 \pm 7,5.2,1706 \pm 105)$, $(699 \pm 21,1.5,1708 \pm 139)$ for dips. Benoit et al. (2003) report the ARCHEOPS first peak location to be at $220 \pm 6$; with our method, all sliders of width $N \geq 4$ detect the first peak accurately; e.g. $N=9$ probing $\ell$ values from 110 to 350 give $(221 \pm 6,5.7,5296 \pm 360)$. The recalibration of ARCHEOPS of table 1 has been applied to $\mathcal{C}_{p k, 1}$, bringing it into good accord with the WMAP and March 2003 numbers.)

\begin{tabular}{|l|lllll|}
\hline & WMAP & Jan02 & Jun02 & Jan03 & Mar03 \\
\hline$\ell_{p k, 1}$ & $221_{-1}^{+1}, 20$ & $219_{-6}^{+6}, 3.5$ & $218_{-6}^{+6}, 3.7$ & $220_{-5}^{+5}, 4.5$ & $221_{-1}^{+1}, 20$ \\
$\ell_{p k, 2}$ & $537_{-12}^{+12}, 2.9$ & $538_{-17}^{+17}, 1.6$ & $536_{-14}^{+14}, 1.8$ & $535_{-12}^{+12}, 2.0$ & $535_{-8}^{+8}, 3.8$ \\
$\ell_{p k, 3}$ & & $\left(805_{-10}^{+10}, 1.5\right)$ & $835_{-16}^{+16}, 2.0$ & $826_{-11}^{+11}, 2.2$ & $823_{-12}^{+12}, 2.0$ \\
$\ell_{p k, 4}$ & & & & $\left(1141_{-13}^{+13}, 2.2\right)$ & $\left(1141_{-13}^{+13}, 2.2\right)$ \\
\hline $\mathcal{C}_{p k, 1}$ & $5386_{-58}^{+58}$ & $5393_{-443}^{+443}$ & $5501_{-392}^{+392}$ & $5414_{-325}^{+325}$ & $5388_{-57}^{+57}$ \\
$\mathcal{C}_{p k, 2}$ & $2326_{-85}^{+85}$ & $2351_{-196}^{+196}$ & $2350_{-164}^{+164}$ & $2364_{-140}^{+140}$ & $2313_{-67}^{+67}$ \\
$\mathcal{C}_{p k, 3}$ & & $2565_{-400}^{+400}$ & $2296_{-256}^{+256}$ & $2490_{-231}^{+231}$ & $2272_{-175}^{+175}$ \\
$\mathcal{C}_{p k, 4}$ & & & & $1279_{-241}^{+241}$ & $1219_{-227}^{+227}$ \\
\hline$\ell_{d i p, 1}$ & $414_{-3}^{+3}, 17$ & $412_{-9}^{+9}, 4.2$ & $414_{-9}^{+9}, 4,2$ & $413_{-7}^{+7}, 5.5$ & $414_{-3}^{+3}, 18$ \\
$\ell_{d i p, 2}$ & $697_{-41}^{+41}, 1.0$ & $697_{-28}^{+28}, 1.1$ & $698_{-27}^{+27}, 1.2$ & $647_{-20}^{+20}, 1.6$ & $690_{-21}^{+21}, 1.7$ \\
$\ell_{d i p, 3}$ & & & $1105_{-36}^{+36}, 2.0$ & $1061_{-50}^{+50}, 1.2$ & $1052_{-31}^{+31}, 1.7$ \\
$\ell_{d i p, 4}$ & & & $\left(1384_{-17}^{+17}, 2.4\right)$ & $\left(1324_{-21}^{+21}, 2.9\right)$ & $\left(1324_{-21}^{+21}, 2.9\right)$ \\
\hline $\mathcal{C}_{d i p k, 1}$ & $1619_{-35}^{+35}$ & $1692_{-143}^{+143}$ & $1689_{-122}^{+122}$ & $1742_{-107}^{+107}$ & $1643_{-32}^{+32}$ \\
$\mathcal{C}_{d i p, 2}$ & $1450_{-234}^{+234}$ & $1889_{-203}^{+203}$ & $1800_{-162}^{+162}$ & $1943_{-132}^{+132}$ & $1768_{-101}^{+101}$ \\
$\mathcal{C}_{d i p, 3}$ & & & $862_{-231}^{+231}$ & $1005_{-218}^{+213}$ & $908_{-187}^{+187}$ \\
$\mathcal{C}_{d i p, 4}$ & & & $115_{-284}^{+284}$ & $468_{-119}^{+119}$ & $448_{-114}^{+114}$ \\
\hline
\end{tabular}


Table 3. Peak/dip locations $\ell_{p k / d i p, j}$ and heights $\mathcal{C}_{p k / \text { dip }, j}$ (in $\mu K^{2}$ ) determined by ensemble-averages over the $\mathcal{C}_{\ell}$ database and the weak prior. (Use of the weak prior allows large movement of peak locations associated with the geometry, hence is preferable to more restrictive priors for this application. These numbers should be contrasted with the 'model-independent' numbers of table 2. The comoving sound speed, $r_{s}$, and damping scale, $R_{D}$, and their associated angular scales, $\pi \ell_{s}$ and $\ell_{D}$, are also shown. Database numbers using this method were given in de Bernardis et al. (2002), Pearson et al. (2003) and Ruhl et al. (2003) and the structural parameters for the June 2002 data were given in Sievers et al. (2003). The results here compare well with those given by Spergel et al. (2003) using the MCMC method: $220 \pm 0.9$ and $535 \pm 2$ for the first two peaks. The WMAP team also give $r_{s}=144 \pm 4 \mathrm{Mpc}$ for power-law $\Lambda \mathrm{CDM}$ models, and $r_{s}=147 \pm 2 \mathrm{Mpc}$ using all of the data and a running index. For $\pi \ell_{s}$ they get $299 \pm 2$ and $301 \pm 1$, respectively. When we do WMAP-only, we get similar values to March 2003 for the peak locations and amplitudes, but with larger errors beyond the third for the peaks that WMAP does not cover - errors more similar to those of the January 2003 data.)

\begin{tabular}{|c|c|c|c|c|c|}
\hline & Jan00 & Jan02 & Jun02 & Jan03 & Mar03 \\
\hline$\pi \ell_{s}$ & $292_{-28}^{+31}$ & $299.7_{-3.5}^{+3.5}$ & $300.7_{-3.5}^{+3.5}$ & $299.6_{-2.7}^{+2.7}$ & $300.2_{-1.0}^{+1.0}$ \\
\hline$r_{s}(\mathrm{Mpc})$ & $115_{-20}^{+24}$ & $144_{-7}^{+7}$ & $144_{-6}^{+6}$ & $144.3_{-4.6}^{+4.8}$ & $145.7_{-2.6}^{+2.7}$ \\
\hline & $1218_{-243}^{+304}$ & $1345_{-18}^{+18}$ & $1348_{-15}^{+15}$ & $1344_{-14}^{+14.0}$ & $1353_{-5.4}^{+5.4}$ \\
\hline$R_{D}(\mathrm{Mpc})$ & $8.8_{-2.2}^{+2.9}$ & $10.2_{-0.48}^{+0.50}$ & $10.2_{-0.39}^{+0.40}$ & $10.2_{-0.35}^{+0.36}$ & $10.3_{-0.20}^{+0.21}$ \\
\hline$\ell_{p k, 1}$ & $226_{-20}^{+22}$ & $220_{-3}^{+3}$ & $219_{-3}^{+3}$ & $220_{-2}^{+2}$ & $220_{-1}^{+1}$ \\
\hline$\ell_{p k, 2}$ & $598_{-122}^{+153}$ & $534_{-12}^{+13}$ & $534_{-12}^{+12}$ & $533_{-13}^{+13}$ & $534_{-2}^{+2}$ \\
\hline$\ell_{p k, 3}$ & $881_{-168}^{+208}$ & $810_{-18}^{+18}$ & $812_{-17}^{+18}$ & $810_{-17}^{+17}$ & $812_{-2}^{+2}$ \\
\hline$\ell_{p k, 4}$ & $1143_{-231}^{+289}$ & $1124_{-28}^{+28}$ & $1127_{-29}^{+29}$ & $1124_{-25}^{+25}$ & $1125_{-4}^{+4}$ \\
\hline$\ell_{p k, 5}$ & $1395_{-337}^{+444}$ & $1420_{-31}^{+31}$ & $1424_{-32}^{+32}$ & $1420_{-27}^{+28}$ & $1423_{-4}^{+4}$ \\
\hline $\mathcal{C}_{p k, 1}$ & $5372_{-611}^{+690}$ & $5442_{-274}^{+288}$ & $5487_{-254}^{+266}$ & $5346_{-171}^{+177}$ & $5551_{-34}^{+35}$ \\
\hline $\mathcal{C}_{p k, 2}$ & $2114_{-984}^{+1841}$ & $2495_{-108}^{+113}$ & $2502_{-94}^{+98}$ & $2483_{-78}^{+80}$ & $2486_{-28}^{+28}$ \\
\hline $\mathcal{C}_{p k, 3}$ & $1372_{-908}^{+2678}$ & $2482_{-187}^{+202}$ & $2478_{-158}^{+169}$ & $2461_{-135}^{+143}$ & $2454_{-54}^{+55}$ \\
\hline $\mathcal{C}_{p k, 4}$ & $836_{-480}^{+1123}$ & $1220_{-96}^{+104}$ & $1203_{-84}^{+90}$ & $1218_{-71}^{+76}$ & $1214_{-24}^{+25}$ \\
\hline $\mathcal{C}_{p k, 5}$ & $498_{-327}^{+949}$ & $811_{-95}^{+108}$ & $792_{-85}^{+95}$ & $809_{-73}^{+81}$ & $808_{-23}^{+24}$ \\
\hline$\ell_{d i p, 1}$ & $393_{-48}^{+54}$ & $412_{-5}^{+5}$ & $413_{-5}^{+5}$ & $412_{-4}^{+4}$ & $409_{-1}^{+1}$ \\
\hline$\ell_{d i p, 2}$ & $507_{-123}^{+162}$ & $671_{-27}^{+28}$ & $673_{-22}^{+23}$ & $670_{-10}^{+10}$ & $672_{-2}^{+2}$ \\
\hline$\ell_{d i p, 3}$ & $830_{-188}^{+242}$ & $1012_{-30}^{+31}$ & $1014_{-27}^{+28}$ & $1009_{-15}^{+15}$ & $1010_{-4}^{+4}$ \\
\hline$\ell_{d i p, 4}$ & $1097_{-244}^{+313}$ & $1306_{-35}^{+30}$ & $1308_{-32}^{+33}$ & $1302_{-18}^{+18}$ & $1305_{-4}^{+4}$ \\
\hline$\overline{\mathcal{C}_{\text {dipk }, 1}}$ & $1932_{-668}^{+1020}$ & $1638_{-74}^{+78}$ & $1637_{-70}^{+70}$ & $1627_{-55}^{+57}$ & $1654_{-15}^{+15}$ \\
\hline $\mathcal{C}_{d i p, 2}$ & $1130_{-634}^{+1453}$ & $1712_{-133}^{+144}$ & $1696_{-112}^{+119}$ & $1707_{-66}^{+68}$ & $1689_{-35}^{+35}$ \\
\hline $\mathcal{C}_{d i p, 3}$ & $709_{-479}^{+1469}$ & $974_{-75}^{+80}$ & $962_{-64}^{+68}$ & $975_{-43}^{+44}$ & $964_{-18}^{+19}$ \\
\hline $\mathcal{C}_{\text {dip }, 4}$ & $557_{-334}^{+834}$ & $668_{-73}^{+82}$ & $656_{-63}^{+70}$ & $670_{-41}^{+43}$ & $662_{-17}^{+18}$ \\
\hline
\end{tabular}



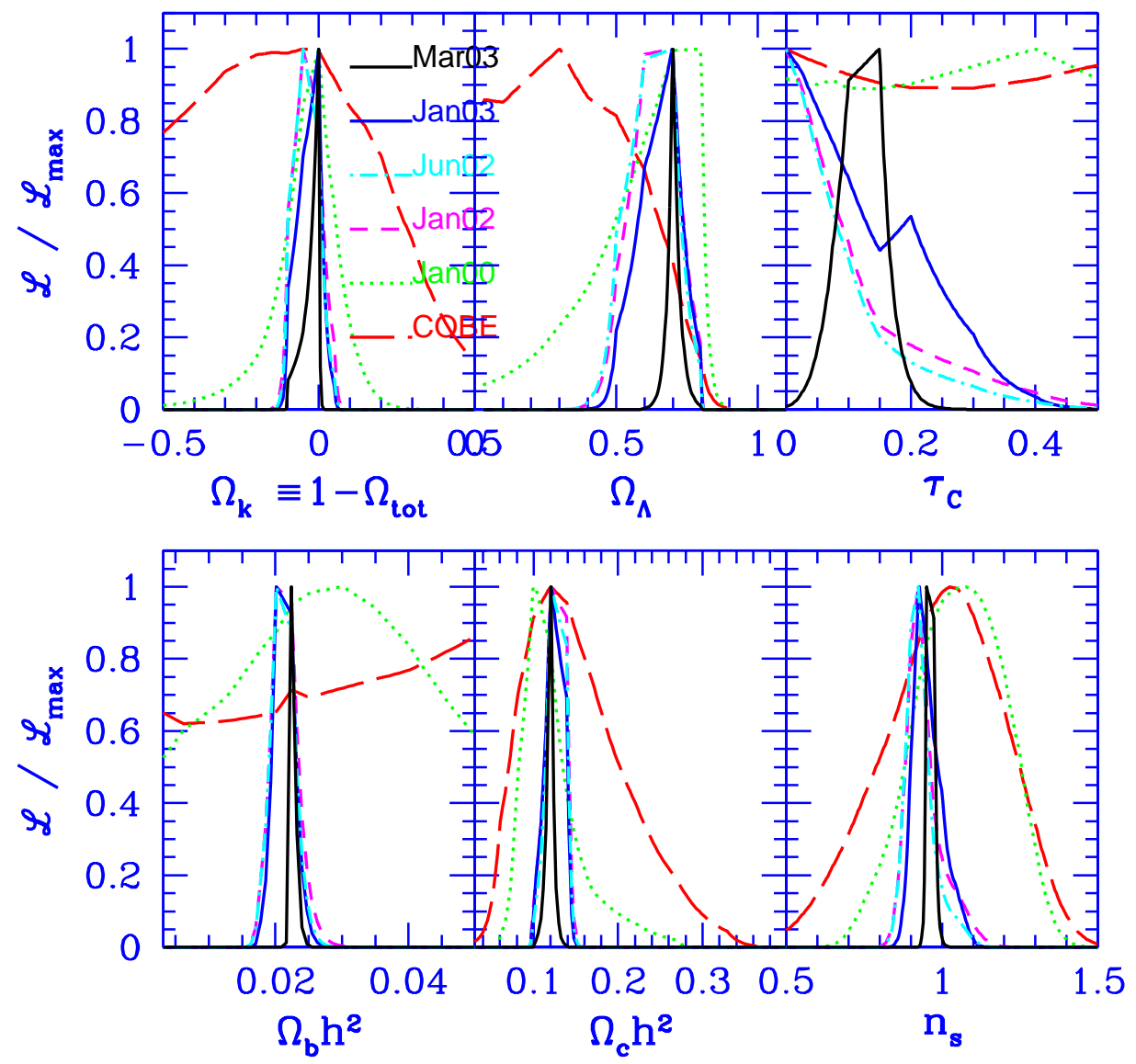

Figure 4. 1D likelihood curves for the weak+flat+LSS prior show that the parameters were in good agreement but also show how much WMAP has sharpened the picture. 


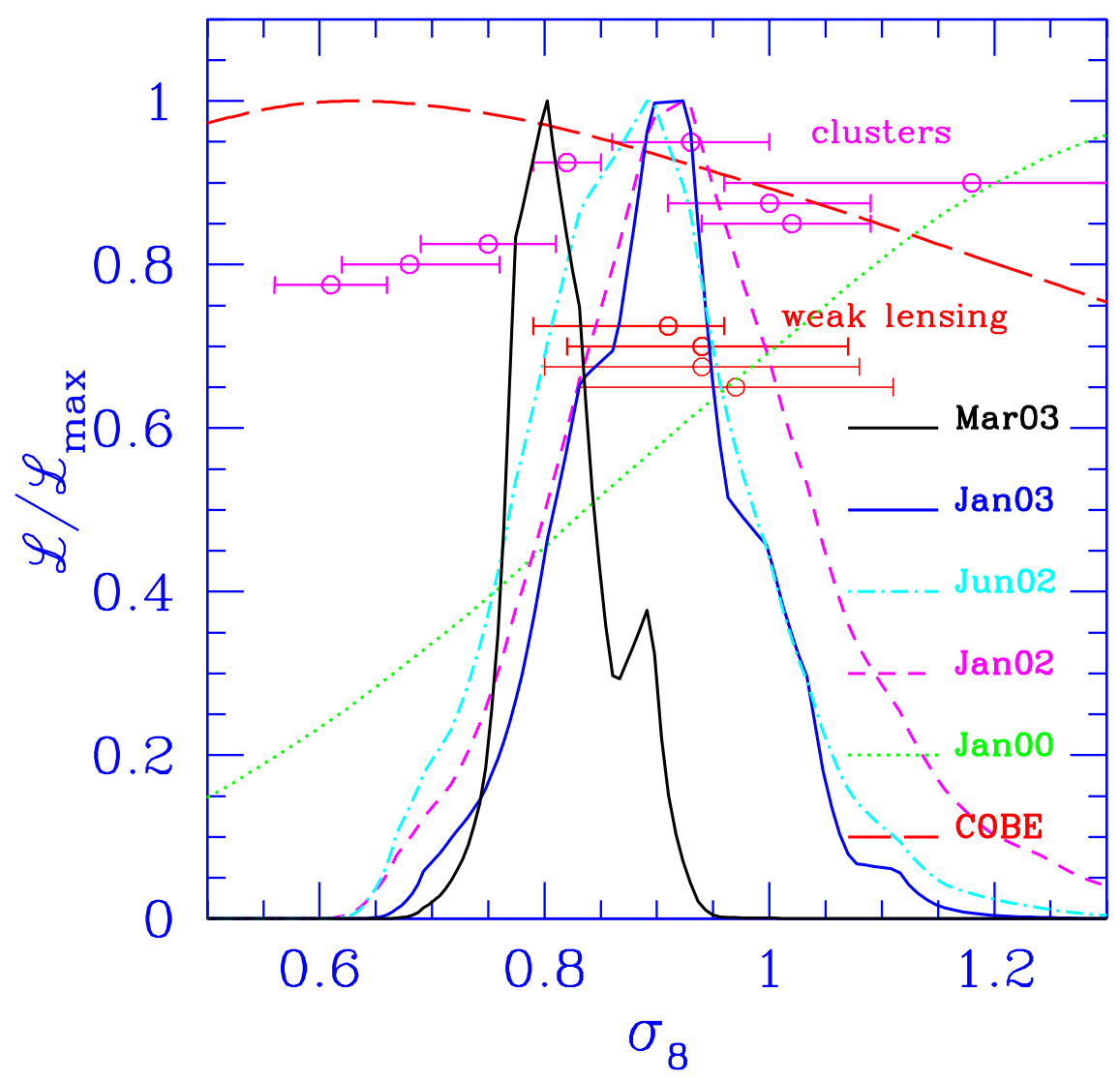

Figure 5. Evolution of $\sigma_{8}$ determinations for the weak+flat prior, with the $\tau_{C}$ prior applied as well for the March 2003 data. These are contrasted with the estimates from weak lensing and clusters which the LSS prior encompasses (Sievers et al. 2003; Bond et al. 2003b). The MCMC results give distributions that extend slightly more to higher $\sigma_{8}$. 
Table 4. Cosmological parameter values from the $\mathcal{C}_{\ell}$ database and their $1 \sigma$ errors are shown, determined after marginalizing over the other six cosmological and the various experimental parameters. (In all cases, the weak prior $(0.45 \leq \mathrm{h} \leq 0.9$, age older than 10 Gyr) is applied, and $w_{Q}$ is fixed at -1 , the cosmological constant case. The first set allow $\Omega_{k}$ to vary, the rest fix it at zero. The LSS prior agrees with weak lensing and redshift survey results, and agrees with most of the cluster determinations. The parameters are very stable if extra 'prior' probabilities for LSS are included, or if the HST range for $h$ is used, or if SN1 data are included. Allowing $w_{Q}$ to vary yields quite similar results. Although the optimal spectra include the January 2000 data in the subsequent mixes in figure 2, these parameter estimates do not. (Their inclusion has little effect.))

\begin{tabular}{|c|c|c|c|c|c|}
\hline database & Jan00 & Jan02 & Jun02 & Jan03 & Mar03 \\
\hline \multicolumn{6}{|l|}{ weak } \\
\hline$\Omega_{t o t}$ & $1.06_{-.10}^{+.16}$ & $1.035_{-.046}^{+.043}$ & $1.038_{-.042}^{+.040}$ & $1.034_{-.036}^{+.039}$ & $1.015_{-.015}^{+.063}$ \\
\hline$\Omega_{b} \mathrm{~h}^{2}$ & $.0339_{-.0246}^{+.0443}$ & $.0222_{-.0021}^{+.0025}$ & $.0221_{-.0020}^{+.0024}$ & $.0221_{-.0018}^{+.0023}$ & $.0233_{-.0013}^{+.0013}$ \\
\hline$\Omega_{c d m} \mathrm{~h}^{2}$ & $.198_{-.080}^{+.088}$ & $.130_{-.028}^{+.031}$ & $.124_{-.025}^{+.026}$ & $.125_{-.022}^{+.021}$ & $.111_{-.010}^{+.010}$ \\
\hline$n_{s}$ & $1.218_{-.163}^{+.135}$ & $0.949_{-.049}^{+.083}$ & $0.938_{-.042}^{+.077}$ & $0.961_{-.047}^{+.081}$ & $0.978_{-.020}^{+.025}$ \\
\hline$\Omega_{\Lambda}$ & $0.34_{-.24}^{+.28}$ & $0.52_{-.20}^{+.17}$ & $0.53_{-.19}^{+.17}$ & $0.57_{-.19}^{+.14}$ & $0.73_{-.10}^{+.06}$ \\
\hline$h$ & $0.60 \pm 0.11$ & $0.56 \pm 0.10$ & $0.55 \pm 0.10$ & $0.57 \pm 0.10$ & $0.68 \pm 0.13$ \\
\hline age & $12.9 \pm 2.1$ & $15.0 \pm 1.4$ & $15.1 \pm 1.3$ & $14.9 \pm 1.2$ & $14.2 \pm 1.3$ \\
\hline$\Omega_{m}$ & $0.72 \pm 0.29$ & $0.53 \pm 0.19$ & $0.51 \pm 0.18$ & $0.49 \pm 0.18$ & $0.33 \pm 0.19$ \\
\hline \multicolumn{6}{|l|}{ flat+weak } \\
\hline$\Omega_{b} \mathrm{~h}^{2}$ & $.0309_{-.0192}^{+.0259}$ & $.0213_{-.0017}^{+.0020}$ & $.0213_{-.0017}^{+.0019}$ & $.0216_{-.0015}^{+.0016}$ & $.0234_{-.0013}^{+.0013}$ \\
\hline$\Omega_{c d m} \mathrm{~h}^{2}$ & $.176_{-.068}^{+.083}$ & $.141_{-.025}^{+.025}$ & $.135_{-.022}^{+.024}$ & $.131_{-.019}^{+.020}$ & $.111_{-.010}^{+.010}$ \\
\hline$n_{s}$ & $1.141_{-.153}^{+.131}$ & $0.931_{-.040}^{+.060}$ & $0.924_{-.036}^{+.050}$ & $0.951_{-.039}^{+.062}$ & $0.979_{-.018}^{+.019}$ \\
\hline$\Omega_{\Lambda}$ & $0.47_{-.30}^{+.23}$ & $0.54_{-.26}^{+.18}$ & $0.58_{-.26}^{+.16}$ & $0.64_{-.16}^{+.11}$ & $0.75_{-.05}^{+.04}$ \\
\hline$h$ & $0.66 \pm 0.12$ & $0.61 \pm 0.10$ & $0.63 \pm 0.10$ & $0.66 \pm 0.09$ & $0.75 \pm 0.05$ \\
\hline age & $12.7 \pm 1.9$ & $13.9 \pm 0.5$ & $14.0 \pm 0.5$ & $13.8 \pm 0.34$ & $13.5 \pm 0.14$ \\
\hline$\Omega_{m}$ & $0.55 \pm 0.24$ & $0.49 \pm 0.20$ & $0.45 \pm 0.20$ & $0.38 \pm 0.15$ & $0.24 \pm 0.05$ \\
\hline$\sigma_{8}$ & $1.23_{-.47}^{+.47}$ & $0.92_{-.12}^{+.12}$ & $0.88_{-.11}^{+.10}$ & $0.90_{-.09}^{+.09}$ & $0.81_{-.04}^{+.06}$ \\
\hline$\Omega_{m} h$ & $0.34 \pm 0.13$ & $0.28 \pm 0.08$ & $0.26 \pm 0.08$ & $0.24 \pm 0.07$ & $0.18 \pm 0.03$ \\
\hline$\Gamma$ & $0.28 \pm 0.12$ & $0.24 \pm 0.08$ & $0.23 \pm 0.07$ & $0.20 \pm 0.06$ & $0.14 \pm 0.03$ \\
\hline \multicolumn{6}{|c|}{ LSS+flat+weak } \\
\hline$\Omega_{b} \mathrm{~h}^{2}$ & $.036_{-.02}^{+.03}$ & $.0217_{-.0018}^{+.0019}$ & $.0215_{-.0017}^{+.0018}$ & $.0217_{-.0015}^{+.0015}$ & $.0228_{-.0013}^{+.0013}$ \\
\hline$\Omega_{c d m} \mathrm{~h}^{2}$ & $.11_{-.03}^{+.04}$ & $.128_{-.012}^{+.011}$ & $.128_{-.011}^{+.011}$ & $.126_{-.012}^{+.012}$ & $.121_{-.010}^{+.010}$ \\
\hline$n_{s}$ & $1.09_{-.16}^{+.15}$ & $0.93_{-.04}^{+.07}$ & $0.93_{-.04}^{+.05}$ & $0.950_{-.037}^{+.067}$ & $0.965_{-.013}^{+.013}$ \\
\hline$\Omega_{\Lambda}$ & $0.61_{-.38}^{+.09}$ & $0.64_{-.09}^{+.08}$ & $0.63_{-.10}^{+.08}$ & $0.66_{-.09}^{+.07}$ & $0.70_{-.05}^{+.05}$ \\
\hline$h$ & $0.67 \pm 0.13$ & $0.65 \pm 0.07$ & $0.65 \pm 0.06$ & $0.66 \pm 0.06$ & $0.69 \pm 0.01$ \\
\hline age & $13.7 \pm 1.9$ & $13.8 \pm 0.5$ & $13.9 \pm 0.5$ & $13.8 \pm 0.28$ & $13.7 \pm 0.03$ \\
\hline$\Omega_{m}$ & $0.40 \pm 0.19$ & $0.36 \pm 0.09$ & $0.37 \pm 0.09$ & $0.35 \pm 0.08$ & $0.30 \pm 0.011$ \\
\hline$\sigma_{8}$ & $0.85_{-.22}^{+.26}$ & $0.88_{-.08}^{+.09}$ & $0.86_{-.07}^{+.08}$ & $0.89_{-.07}^{+.06}$ & $0.86_{-.04}^{+.04}$ \\
\hline$\Omega_{m} h$ & $0.25 \pm 0.10$ & $0.23 \pm 0.04$ & $0.24 \pm 0.04$ & $0.23 \pm 0.04$ & $0.206 \pm 0.006$ \\
\hline$\Gamma$ & $0.18 \pm 0.05$ & $0.20 \pm 0.04$ & $0.20 \pm 0.04$ & $0.19 \pm 0.033$ & $0.174 \pm 0.006$ \\
\hline
\end{tabular}


Table 5. MCMC determinations of cosmic parameters should be compared with those of the $\mathcal{C}_{\ell}$ database given in table 4 . (Means and standard deviations are given here, rather than the Bayesian 50\%, with $16 \%$ and $84 \%$ ' $1 \sigma$ ' errors. In addition, for the March 2003 data, the Bayesian results using the 899 WMAP points in the database with the $\tau_{C}$-prior are shown in the last column. Note how $\sigma_{8}$ increases when the logarithmic running of the spectral slope is allowed, a manifestation of the high correlation of $\sigma_{8}$ and $\tau_{C}$ with $d n_{s} / d \ln k$ as well as $n_{s}$. The values are therefore sensitive to the priors imposed.)

\begin{tabular}{|c|c|c|c|c|c|}
\hline MCMC & Jan02 & Jun02 & Jan03 & Mar03 & Mar03(899db) \\
\hline \multicolumn{6}{|l|}{ weak } \\
\hline$\Omega_{t o t}$ & $1.024_{-.041}^{+.041}$ & $1.030_{-.039}^{+.039}$ & $1.039_{-.037}^{+.037}$ & $1.050_{-.032}^{+.032}$ & $1.016_{-.03}^{+.08}$ \\
\hline$\Omega_{b} \mathrm{~h}^{2}$ & $.0215_{-.0017}^{+.041}$ & $.0212_{-.0015}^{+.0015}$ & $.0214_{-. .0013}^{+.0013}$ & $.0222_{-.0007}^{+.032}$ & $.0227_{-.0013}^{+.003}$ \\
\hline$\Omega_{c d m} \mathrm{~h}^{2}$ & $.145_{-.026}^{+.026}$ & $.135_{-.023}^{+.023}$ & $.131_{-.017}^{+.017}$ & $.120_{-.009}^{+.009}$ & $.112_{-.010}^{+.010}$ \\
\hline$n_{s}$ & $0.995_{-.063}^{+.063}$ & $0.976_{-.053}^{+.053}$ & $0.965_{-.039}^{+.039}$ & $0.950_{-.017}^{+.017}$ & $0.960_{-.013}^{+.016}$ \\
\hline$\Omega_{\Lambda}$ & $0.45_{-.18}^{+.18}$ & $0.48^{0}+.16_{-.16}$ & $0.51_{-.15}^{+.15}$ & $0.55_{-.11}^{+.11}$ & $0.71_{-.30}^{+.06}$ \\
\hline$h$ & $0.56 \pm 0.11$ & $0.55 \pm 0.10$ & $0.56 \pm 0.11$ & $0.55 \pm 0.09$ & $0.61 \pm 0.14$ \\
\hline age & $14.4 \pm 1.3$ & $14.9 \pm 1.3$ & $14.9 \pm 1.2$ & $15.3 \pm 1.0$ & $14.8 \pm 1.5$ \\
\hline$\Omega_{m}$ & $0.58 \pm 0.19$ & $0.55 \pm 0.17$ & $0.54 \pm 0.18$ & $0.51 \pm 0.14$ & $0.45 \pm 0.22$ \\
\hline \multicolumn{6}{|l|}{ flat+weak } \\
\hline$\overline{\Omega_{b} \mathrm{~h}^{2}}$ & $.0226_{-.0026}^{+.0026}$ & $.0219_{-.0024}^{+.0024}$ & $.0219_{-.0018}^{+.0018}$ & $.0230_{-.0011}^{+.0011}$ & $.0228_{-.0013}^{+.0013}$ \\
\hline$\Omega_{c d m} \mathrm{~h}^{2}$ & $.140_{-.026}^{+.026}$ & $.132_{-.024}^{+.024}$ & $.128_{-.018}^{+.018}$ & $.117_{-.010}^{+.010}$ & $.116_{-.010}^{+.010}$ \\
\hline$n_{s}$ & $1.02_{-.087}^{+.086}$ & $0.994_{-.073}^{+.024}$ & $0.973_{-.045}^{+.018}$ & $0.967_{-.029}^{+.029}$ & $0.965_{-.013}^{+.010}$ \\
\hline$\Omega_{\Lambda}$ & $0.56_{-.20}^{+.20}$ & $0.58_{-.19}^{+.19}$ & $0.65_{-.13}^{+.13}$ & $0.72_{-.05}^{+.05}$ & $0.73_{-.05}^{+.05}$ \\
\hline$h$ & $0.64 \pm 0.11$ & $0.64 \pm 0.10$ & $0.67 \pm 0.08$ & $0.71 \pm 0.05$ & $0.72 \pm 0.05$ \\
\hline age & $13.6 \pm 0.6$ & $13.9 \pm 0.5$ & $13.6 \pm 0.40$ & $13.6 \pm 0.22$ & $13.6 \pm 0.12$ \\
\hline$\Omega_{m}$ & $0.44 \pm 0.20$ & $0.42 \pm 0.19$ & $0.35 \pm 0.13$ & $0.28 \pm 0.05$ & $0.27 \pm 0.05$ \\
\hline$\sigma_{8}$ & $1.05_{-.20}^{+.20}$ & $0.94_{-.13}^{+.13}$ & $0.89_{-.09}^{+.09}$ & $0.85_{-.06}^{+.06}$ & $0.83_{-.06}^{+.05}$ \\
\hline flat+weak & \multicolumn{5}{|l|}{$\bar{~} d n_{s} / d \ln k$} \\
\hline$\Omega_{b} \mathrm{~h}^{2}$ & $.0211_{-.0027}^{+.0027}$ & $.0207_{-.0024}^{+.0024}$ & $.0207_{-.0020}^{+.0020}$ & $.0229_{-.0018}^{+.0018}$ & \\
\hline$\Omega_{c d m} \mathrm{~h}^{2}$ & $.154_{-.028}^{+.028}$ & $.147_{-.026}^{+.026}$ & $.149_{-.025}^{+.025}$ & $.121_{-.016}^{+.016}$ & \\
\hline$n_{s}\left(k_{n}\right)$ & $0.920_{-.119}^{+.119}$ & $0.897_{-.101}^{+.1026}$ & $0.874_{-.075}^{+.075}$ & $0.924_{-.059}^{+.059}$ & \\
\hline$-d n_{s}\left(k_{n}\right) / d \ln k$ & $0.098_{-.060}^{+.060}$ & $0.101_{-.056}^{+.056}$ & $0.091_{-.045}^{+.045}$ & $0.083_{-.033}^{+.033}$ & \\
\hline$\Omega_{\Lambda}$ & $0.44_{-.23}^{+.23}$ & $0.45_{-.23}^{+.23}$ & $0.48_{-.21}^{+.21}$ & $0.70_{-.10}^{+.10}$ & \\
\hline$h$ & $0.59 \pm 0.10$ & $0.58 \pm 0.10$ & $0.60 \pm 0.10$ & $0.71 \pm 0.08$ & \\
\hline age & $13.8 \pm 0.6$ & $14.0 \pm 0.5$ & $13.8 \pm 0.42$ & $13.5 \pm 0.36$ & \\
\hline$\Omega_{m}$ & $0.56 \pm 0.23$ & $0.55 \pm 0.23$ & $0.52 \pm 0.21$ & $0.30 \pm 0.10$ & \\
\hline$\sigma_{8}$ & $1.20_{-.24}^{+.24}$ & $1.13_{-22}^{+.22}$ & $0.99_{-13}^{+.13}$ & $0.96_{-08}^{+.08}$ & \\
\hline
\end{tabular}

